\title{
NMDA Receptors Enhance the Fidelity of Synaptic Integration
}

\author{
Chenguang Li and ${ }^{\circledR}$ Allan T. Gulledge
}

https://doi.org/10.1523/ENEURO.0396-20.2020

Department of Molecular and Systems Biology, Geisel School of Medicine at Dartmouth College, Hanover, NH 03755

\begin{abstract}
Excitatory synaptic transmission in many neurons is mediated by two coexpressed ionotropic glutamate receptor subtypes, AMPA and NMDA receptors, that differ in kinetics, ion selectivity, and voltage-sensitivity. AMPA receptors have fast kinetics and are voltage-insensitive, while NMDA receptors have slower kinetics and increased conductance at depolarized membrane potentials. Here, we report that the voltage dependency and kinetics of NMDA receptors act synergistically to stabilize synaptic integration of EPSPs across spatial and voltage domains. Simulations of synaptic integration in simplified and morphologically realistic dendritic trees revealed that the combined presence of AMPA and NMDA conductances reduce the variability of somatic responses to spatiotemporal patterns of excitatory synaptic input presented at different initial membrane potentials and/or in different dendritic domains. This moderating effect of the NMDA conductance on synaptic integration was robust across a wide range of AMPA-to-NMDA ratios, and results from synergistic interaction of NMDA kinetics (which reduces variability across membrane potential) and voltage dependence (which favors stabilization across dendritic location). When combined with AMPA conductance, the NMDA conductance compensates for voltage-dependent and impedance-dependent changes in synaptic driving force, and distance-dependent attenuation of synaptic potentials arriving at the axon, to increase the fidelity of synaptic integration and EPSPspike coupling across both neuron state (i.e., initial membrane potential) and dendritic location of synaptic input. Thus, synaptic NMDA receptors convey advantages for synaptic integration that are independent of, but fully compatible with, their importance for coincidence detection and synaptic plasticity.
\end{abstract}

Key words: AMPA receptor; dendrite; EPSP-spike coupling; NMDA receptor; synapse; synaptic integration

\section{Significance Statement}

Glutamate is an excitatory neurotransmitter that, at many synapses, gates two coexpressed receptor subtypes (AMPA and NMDA receptors). Computational simulations reveal that the combined synaptic presence of AMPA and NMDA receptors reduces variability in synaptic integration in response to identical patterns of synaptic input delivered to different dendritic locations and/or at different initial membrane potentials. This results from synergistic interaction of the slower kinetics and voltage dependence of NMDA receptors, which combine to enhance synaptic currents when synaptic driving forces are otherwise reduced (e.g., at depolarized membrane potentials or in distal, high-impedance dendrites). By stabilizing synaptic integration across dendritic location and initial membrane potential, NMDA receptors provide advantages independent of, but fully compatible with, their well-known contribution to synaptic plasticity. 


\section{Introduction}

In the vertebrate central nervous system, fast excitatory synaptic transmission is mediated primarily by the amino acid glutamate, which at many synapses gates two coexpressed ionotropic receptors: AMPA and NMDA receptors. While both receptor subtypes are gated by glutamate, and are permeable to monovalent cations, NMDA receptors are distinct in having slower kinetics, permeability for calcium, and voltage dependence because of channel blockade by extracellular magnesium ions at hyperpolarized membrane potentials (for review, see lacobucci and Popescu, 2017). These features of NMDA receptors, which are highly conserved across phyla (Greer et al., 2017), are critical for their well-established role in gating associative synaptic plasticity, including certain types of long-term potentiation and depression (for review, see Lüscher and Malenka, 2012). NMDA receptors also influence synaptic integration, as the voltage dependence of NMDA receptors can promote linear (Cash and Yuste, 1998) or supralinear (Schiller et al., 2000) summation of EPSPs that occur in sufficient spatial and temporal proximity to relieve magnesium block of NMDA channels, and can reduce the voltage-dependent variability of mixed AMPA/NMDA synaptic currents (Diamond and Copenhagen, 1993; Connelly et al., 2016).

Because the input impedance of dendrites increases with distance from the soma, distal synapses generate larger, more depolarizing local dendritic EPSPs than do proximal synapses (Jaffe and Carnevale, 1999; Gulledge et al., 2005; Lajeunesse et al., 2013). Yet the ability of an individual AMPA-mediated EPSP to recruit synaptic NMDA conductance is tempered by its rapid kinetics ( $\sim 0.2-$ and 2.0-ms activation and decay, respectively), which, when combined with the limited local capacitance of narrow dendrites and dendritic spines, generates EPSPs that decay too rapidly to efficiently recruit slower activating ( $>2 \mathrm{~ms}$ ) NMDA conductances (Stern et al., 1992; Gulledge et al., 2012). Instead, network-driven patterns of synaptic input interact within the dendritic tree based on their spatiotemporal relationships to recruit NMDA conductances (Polsky et al., 2009; Grienberger et al., 2014; Palmer et al., 2014; Cichon and Gan, 2015), especially when they occur in high-impedance dendritic branches or spines (Branco and Häusser, 2011; Gulledge et al., 2012; Harnett et al., 2012). NMDA conductances are also

This work was supported by the National Institute for Mental Health Grant R01 MH099054 (A.T.G.), a Frank and Myra Weiser Scholar Award (A.T.G.), and the Kaminsky Fund for Undergraduate Research at Dartmouth College (C.L.).

Acknowledgements: We thank Arielle Baker, Nik Dembrow, and Greg Stuart for comments and suggestions on a draft of this manuscript and Saiko Ikeda for assistance with data analyses.

Correspondence should be addressed to Allan T. Gulledge at allan@ dartmouth.edu.

https://doi.org/10.1523/ENEURO.0396-20.2020

Copyright @ $2021 \mathrm{Li}$ and Gulledge

This is an open-access article distributed under the terms of the Creative Commons Attribution 4.0 International license, which permits unrestricted use, distribution and reproduction in any medium provided that the original work is properly attributed. recruited when barrages involve repetitive activation of the same synapses, as the extended occupancy of glutamate sites on NMDA receptors ( $100 \mathrm{~ms})$ "prime" them for immediate gating during subsequent AMPAmediated local depolarization (Schiller et al., 2000; Polsky et al., 2004). With sufficient levels of synaptic activation, inward NMDA currents become self-sustaining "NMDA spikes" that amplify and prolong synaptic depolarization to generate supralinear summative events at the soma and axon (for review, see Antic et al., 2010; Branco and Häusser, 2010; Major et al., 2013; Grienberger et al., 2015).

While most prior studies examining the impact of synaptic conductance on the location and/or voltage dependence of synaptic transmission have focused on single synaptic events (Jaffe and Carnevale, 1999; Lajeunesse et al., 2013), we reasoned that the voltage dependency of NMDA receptors, combined with the distance-dependent electrotonic structure of dendritic trees, should reduce the variability of somatic drive (as measured as somatic depolarization and/or action potential generation) in response to spatiotemporal patterns of synaptic input occurring at different dendritic locations and/or from different initial membrane potential states. Here, we test this hypothesis using computational simulations to compare the impact of synaptic conductance (AMPA-only, NMDA-only, or both conductances together) on the integration of patterns of afferent input delivered to different dendritic locations and/ or at different initial membrane potentials. Our results reveal that AMPA and NMDA conductances interact in a complementary fashion to stabilize synaptic integration and EPSP-spike coupling in ways that are independent of, yet fully compatible with, their well-established roles in coincidence detection and synaptic plasticity.

\section{Materials and Methods}

\section{Code accessibility}

The code used to generate the data in this paper is freely available online at http://modeldb.yale.edu/266802. The code is also available as Extended Data 1.

\section{Computational models}

Simulations were made using NEURON 7.7 software (Carnevale and Hines, 2006; RRID: SCR_005393) run on MacOS 10.14 and/or on the Neuroscience Gateway portal (Sivagnanam et al., 2013). Morphologies used include "ball-and-stick" model neurons, consisting of somata connected to spinous dendrites of variable length, a hippocampal CA3 pyramidal neuron (Hemond et al., 2009), and a hippocampal dentate granule neuron (SchmidtHieber et al., 2007). Table 1 lists the dimensions and membrane parameters for all neuron morphologies. Active mechanisms consisted primarily of fast-inactivating voltage-gated sodium and delayed-rectifier potassium conductances (source codes available in ModelDB, entry 144385). Spines with neck resistances of $500 \mathrm{M} \Omega$ (Harnett et al., 2012) were positioned at 1- $\mu \mathrm{m}$ intervals along dendrites. Axons in all models were $2000 \mu \mathrm{m}$ long, 
Table 1: Model parameters

\begin{tabular}{|c|c|c|c|c|}
\hline Neuron morphology & Compartment & Dimensions $(\mathrm{I} \times \mathrm{w})$ & $\begin{array}{l}\text { Number of } \\
\text { segments }\end{array}$ & Active properties (max. conductance) \\
\hline $\begin{array}{l}\mathrm{CA}^{*} \text { and dentate granule } \\
\text { neurons }\end{array}$ & Soma & As reconstructed & 3 & $\begin{array}{l}\mathrm{Na}^{+}: 100 \mathrm{pS} / \mu \mathrm{m}^{2} \\
\mathrm{~K}^{+}: 100 \mathrm{pS} / \mu \mathrm{m}^{2}\end{array}$ \\
\hline \multirow[t]{3}{*}{ All morphologies } & Spines & $\begin{array}{l}\text { Neck: } 1 \times \sim 0.05 \mu \mathrm{m} \\
\text { Head: } 0.5 \times 0.5 \mu \mathrm{m}\end{array}$ & $\begin{array}{l}1 \\
1\end{array}$ & As in parent dendritic compartment \\
\hline & AIS & $\begin{array}{l}40 \mu \mathrm{m} \text {, tapering from } 2 \text { to } \\
0.5 \mu \mathrm{m}\end{array}$ & 40 & $\begin{array}{l}\mathrm{Na}^{+}: 100 \mathrm{pS} / \mu \mathrm{m}^{2}(1 \mathrm{st} 5 \mu \mathrm{m}) \text { or } 8000 \mathrm{pS} / \mu \mathrm{m}^{2} \\
\mathrm{~K}^{+}: 100 \mathrm{pS} / \mu \mathrm{m}^{2}(1 \mathrm{st} 5 \mu \mathrm{m}) \text { or } 2000 \mathrm{pS} / \mu \mathrm{m}^{2}\end{array}$ \\
\hline & Axon & $2000 \times 0.5 \mu \mathrm{m}$ & 201 & $\begin{array}{l}\mathrm{Na}^{+}: 300 \mathrm{pS} / \mu \mathrm{m}^{2} \\
\mathrm{~K}^{+}: 60 \mathrm{pS} / \mu \mathrm{m}^{2}\end{array}$ \\
\hline
\end{tabular}

Listed are the parameters for model neurons used in this study. General model parameters: $R_{M}=15 \mathrm{k} \Omega \bullet \mathrm{cm}^{2} ; \mathrm{C}_{\mathrm{M}}=1 \mu \mathrm{F} / \mathrm{cm}^{2} ; \mathrm{R}_{\mathrm{i}}=100 \Omega \bullet \mathrm{cm} ; \mathrm{E}_{\mathrm{pas}}=-55$ to $-85 \mathrm{mV}$, as indicated in text; time steps, 10 or $25 \mu \mathrm{s}$; nominal temperature $37^{\circ} \mathrm{C}$. ${ }^{*}$ The ball-and-stick model in Figure 1 and the $\mathrm{CA} 3$ neuron in Figure 8 are purely passive (including in somata and axons).

had diameters of $0.5 \mu \mathrm{m}$, and were attached to the soma via a $40-\mu \mathrm{m}$ axon initial segment (AIS) that tapered from $2.0 \mu \mathrm{m}$ (at the soma) to $0.5 \mu \mathrm{m}$ (at the axon). Except for the AIS, voltage-gated conductances were evenly distributed in axons (i.e., axons were modeled as unmyelinated; see Table 1 for sodium and potassium channel densities in each neuronal compartment). Unless otherwise noted, ball-and-stick neurons included somata (20 $\times 10 \mu \mathrm{m})$ attached to a single tapering $(5 \mu \mathrm{m}$ to $1 \mu \mathrm{m})$ dendrite of variable length $(0.2-1 \mathrm{~mm}$; input resistances $\left[R_{N}\right]$ ranging from $\sim 125$ to $\sim 310 \mathrm{M} \Omega$ ). Because $R_{N}$ can influence the impact of dendritic location on synaptic integration, for some simulations we delivered synaptic input to a $200-\mu \mathrm{m}$ dendrite attached to a larger neuron typically having three additional $600-\mu \mathrm{m}$ dendrites that did not receive synaptic input. In all simulations, models were initiated following a 1-s passive run to allow active conductances to reach a steady state. Simulations were run with time steps of 10 or $25 \mu$ s at a nominal temperature of $37^{\circ} \mathrm{C}$.

\section{Simulated synaptic inputs}

In most simulations, synaptic conductances were located on spine heads. AMPA conductances (ModelDB entry 120798) typically had exponential rise and decay time constants of 0.2 and $2 \mathrm{~ms}$, respectively, a reversal potential of $0 \mathrm{mV}$, and a maximum conductance of 500 pS, unless otherwise noted. NMDA inputs were based on the AMPA conductance, with added voltage dependence (as in Model DB entry 184725), and unless otherwise noted had nominal maximum conductances of $1 \mathrm{nS}$ (typically reaching $\sim 46 \mathrm{pS}$ during individual AMPA+NMDA synaptic events occurring from a membrane potential of $-55 \mathrm{mV}$; see also Figs. $1 B, 4 A)$, reversal potentials of $+5 \mathrm{mV}$, and exponential rise and decay time constants of 3 and $90 \mathrm{~ms}$, respectively. Single synaptic potentials were monitored at the soma and at the dendritic site of synaptic input. For barrages of synaptic input, voltage responses were measured at the soma and at the mid-point of the dendritic span receiving synaptic input. During spike threshold tests, synaptic thresholds were determined by monitoring action potentials in the midpoint of the axon. During non-spike threshold tests, synaptic thresholds were determined as somatic events reaching $5 \mathrm{mV}$ (for the dentate granule cell) or $3 \mathrm{mV}$ (for the CA3 neuron) above the resting membrane potential (RMP).

Synaptic barrages of $n$ synaptic activations, delivered to discrete dendritic compartments, were defined by sampling two random variables $n$ times for a set of $n$ pairs. The first random variable in the pair determined the site of the $n$th synaptic input and was chosen from a uniform distribution spanning integers 0-49 (for a 50$\mu \mathrm{m}$ range), which was then applied to the dendritic segment of interest (i.e., from 620 to $669 \mu \mathrm{m}$ from the soma). The second random variable, which determined synaptic timing, was chosen from a Gaussian distribution (width of $50 \mathrm{~ms}$ ). Each pattern maintained a single ordered set of sampled pairs. The size of the set varied depending on the number $\mathrm{n}$, but the content and order of the pairs did not change for a given pattern. For instance, within one pattern, barrages with $\mathrm{n}$ and $n+1$ synaptic activations were identical except for the $n+1$ th additional activation. These spatiotemporal patterns of synaptic input were iteratively moved along dendrites at 10- or 20- $\mu \mathrm{m}$ intervals to compare the effect of input location on local and somatic synaptic responses. Ten different randomized spatiotemporal patterns of synaptic input were used to determine the stability of EPSP-spike coupling over input pattern, dendritic location, and RMP. Nominal RMPs were set by uniformly adjusting the reversal potential for the passive leak conductance in all model compartments.

Variation of EPSP-spike coupling and somatic drive was quantified as coefficient of variation ( $C V$; SD normalized by mean), which provides a relative measure of variability in synaptic thresholds across all dendritic 
A
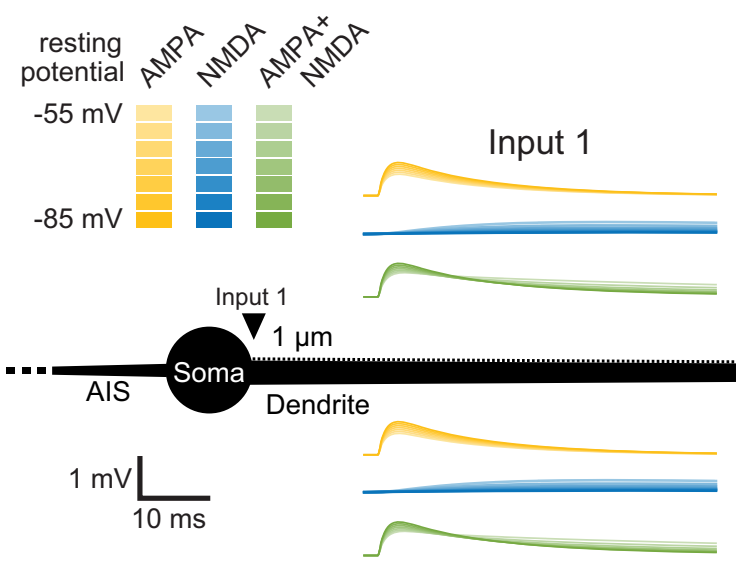

B

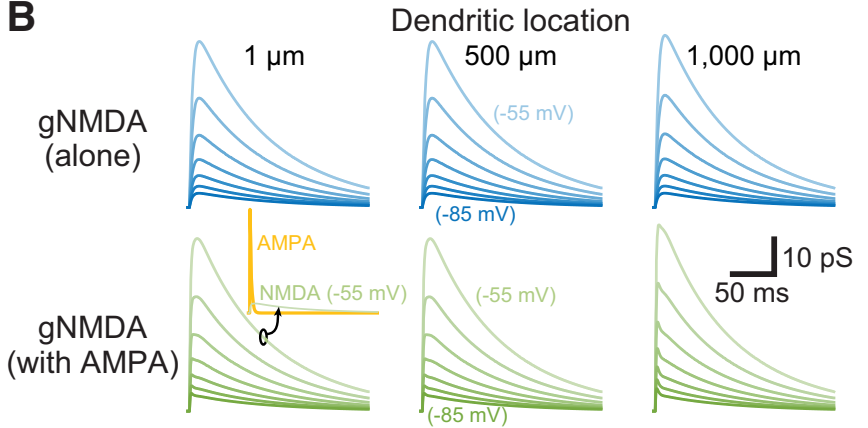

Recorded locally in the dendrite

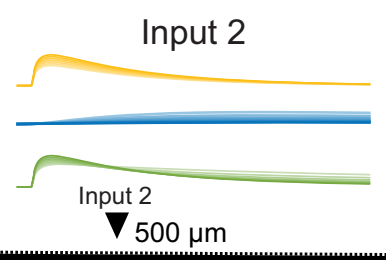

Recorded at soma

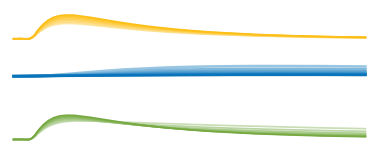

C

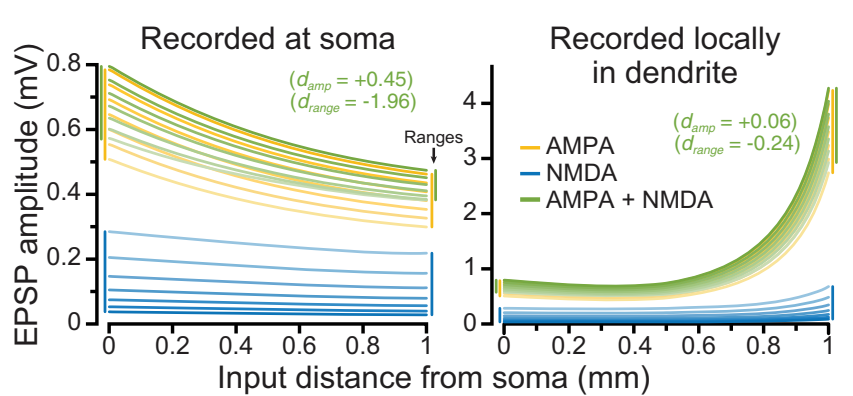

Figure 1. Impact of membrane potential and dendritic location on synaptic conductances and their resulting EPSPs. $\boldsymbol{A}$, Individual synaptic inputs were activated along the dendrite $(1000 \mu \mathrm{m}$ in length) of a passive ball-and-stick model neuron (black; not to scale). Shown are somatic (bottom) and local dendritic (top) EPSPs generated by synapses placed at three dendritic locations (1, 500, and $1000 \mu \mathrm{m}$ from the soma), at seven different RMPs ( -55 to $-85 \mathrm{mV}$, as indicated by color depth). AMPA-only EPSPs are shown in yellow, NMDA-only synapses are shown in blue, and inputs having both AMPA and NMDA conductance are shown in green. $\boldsymbol{B}$, The NMDA conductances underlying the EPSPs shown in $\boldsymbol{A}$. Inset shows the 500 pS AMPA (yellow) and NMDA (green) conductances for the AMPA+NMDA input at $-55 \mathrm{mV}$ at the most proximal dendritic location (shown to scale). C, Plots of the amplitudes of EPSPs having the indicated synaptic conductances measured at the soma (left) or locally at the site of synaptic input (right), versus dendritic location of the synapse. Synaptic conductances are color coded as in $\boldsymbol{A}$ for seven RMPs from -85 to $-55 \mathrm{mV}$, at 5 - $\mathrm{mV}$ intervals. Inset shows the effect sizes (d) for AMPA+NMDA EPSP amplitudes (across all dendritic locations and RMPs) and ranges (across all RMPs) relative to AMPA-only EPSPs. Colored vertical bars at margins indicate the ranges of amplitudes across all RMPs for the most proximal and most distal inputs.

locations and/or membrane potentials independent of response magnitudes (as opposed to measures of absolute range or SD, which are difficult to compare across models having different mean thresholds, and which may overestimate effective variability if functions [e.g., vs distance or voltage] are nonlinear). In the longest dendrites tested (the 1000- $\mu \mathrm{m}$ ball-and-stick and CA3 neurons), inputs to distal dendritic locations became so remote that threshold numbers of synaptic inputs rose exponentially to infinite values (i.e., beyond our maximum tested value of 10,000 ), as at very distal distances even voltage-clamping a $50-\mu \mathrm{m}$ dendritic segment to $0 \mathrm{mV}$ would fail to generate action potentials in the axon. Trials with AMPA-only inputs always hit this limit earliest (i.e., at slightly more proximal dendritic locations than other conductances), and so CVs for all conductances (AMPA-only, NMDA-only, or both AMPA and NMDA) were calculated only across dendritic locations where AMPA synaptic thresholds were measurable.

\section{Statistical analyses}

Results are reported as mean \pm SD. Because our simulations are deterministic, we know a priori that differences in synaptic responses are "real" (that is to say, we know they result from the changes in synaptic conductance coded into the simulation). To quantify the relative impact of synaptic conductance on the variability of synaptic integration (as measured by $\mathrm{CV}$ of synaptic thresholds) across RMPs and/ or dendritic locations, we calculated the effect size (d) for each synaptic conductance as the mean difference in $\mathrm{CV}$ (relative to AMPA-only trials) normalized by the SD (s) of the AMPA-only trials, according to the following formula:

$$
d=\frac{\left(\bar{x}_{\text {test }}-\bar{x}_{\text {AMPA-only }}\right)}{S_{\text {AMPA-only }}},
$$

where $\bar{x}_{\text {AMPA-only }}$ and $\bar{x}_{\text {test }}$ are the means of CVs of synaptic thresholds calculated across trials (e.g., across patterns of synaptic input, dendritic locations, and/or RMPs) for AMPA-only simulations and those with modified 
synaptic conductances, respectively, and sAMPA-only is the SD for CVs in AMPA-only trials. Thus, $d$ represents both the direction (by sign) and magnitude (in units of $S_{\text {AMPA-only }}$ ) of differences in mean variability of synaptic thresholds. This generates a more conservative measure of effect size than other approaches (such as Cohen's $d$ ) because the SD of synaptic thresholds for AMPA+NMDA trials was typically much smaller than for AMPA-only trials (see, for instance, the error bars in Figs. 2D, 6C). Quantifying effect size in this manner allows direct comparison of the relative impact of synaptic conductance on variability in neurons of different sizes and across different RMPs.

\section{Results}

\section{Effect of synaptic conductance type on individual EPSPs}

We first compared the relative efficacy of individual synapses consisting of AMPA-only, NMDA-only, or both (AMPA and NMDA) conductances in depolarizing the soma of a simplified and fully passive ball-and-stick model neuron (see Materials and Methods) with the RMP set to one of seven values ( -85 to $-55 \mathrm{mV}$; see Materials and Methods). As expected, given the cable properties of dendrites (Gulledge et al., 2005), the amplitude and kinetics of the resulting EPSPs depended on synaptic conductance type, RMP, and the dendritic location of synaptic input (Fig. 1A). NMDA conductances were larger at depolarized RMPs and/or in distal dendritic compartments (Fig. 1B), generating NMDA-mediated EPSPs that were largest under those conditions (Fig. 1C, light-blue plots). On the other hand, AMPA-only and AMPA+NMDA EPSPs had maximal amplitudes at the most hyperpolarized RMPs, where driving force was greatest (Fig. 1C, dark yellow and dark green plots). Across all dendritic locations and RMPs, somatic EPSP amplitudes averaged $0.48 \pm 0.10$, $0.11 \pm 0.07$, and $0.52 \pm 0.09 \mathrm{mV}$ for AMPA-only, NMDAonly, and AMPA+NMDA responses, respectively. Somatic EPSPs generated by AMPA+NMDA conductances were larger (effect size, $d$, of +0.45 relative to AMPA-only inputs) and less variable across dendritic locations and RMPs $(C V=0.18)$ than EPSPs generated by AMPA-only ( $C V=0.22)$ or NMDA-only $(C V=0.65)$ inputs. Similarly, AMPA+NMDA somatic EPSPs had smaller absolute amplitude ranges across RMPs for each dendritic location (mean range of $0.14 \pm 0.04 \mathrm{mV}$; $d=-1.96$ relative to AMPA-only inputs) relative to ranges for AMPAonly $(0.21 \pm 0.03 \mathrm{mV})$ or NMDA-only $(0.21 \pm 0.02 \mathrm{mV})$ EPSPs. While somatic EPSP amplitudes decremented with synaptic distance from the soma, amplitudes at the site of synaptic input became progressively larger at distal dendritic locations (Fig. 1C), and averaged $0.96 \pm 0.68$, $0.15 \pm 0.11$, and $1.00 \pm 0.69 \mathrm{mV}$ for AMPA-only, NMDAonly, and AMPA+NMDA inputs, respectively. AMPA+NMDA dendritic EPSPs were marginally larger than AMPA-only EPSPs $(d=+0.06)$ and had slightly smaller absolute ranges across RMPs $(d=-0.24)$. These data demonstrate that adding an NMDA conductance to individual AMPA-only EPSPs generates marginally larger EPSPs with slightly reduced variability across dendritic location and RMP.

\section{Impact of synaptic conductance on EPSP-spike coupling}

Although the impact of the NMDA conductance on somatic EPSP variability was small for individual EPSPs (Fig. 1A, compare yellow and green traces), we reasoned that its impact might be amplified when dendrites experience barrages of excitatory synaptic input. To test this, we measured EPSP-spike coupling in ball-and-stick model neurons with dendrites of various lengths (200$1000 \mu \mathrm{m})$ and compared, across dendritic locations and RMPs, the threshold number of synaptic activations necessary for action potential initiation in the axon. Figure $2 \mathrm{~A}$ shows results for the most proximal dendritic location in a model neuron with a 1000- $\mu$ m dendrite with a RMP of $-70 \mathrm{mV}$. In each model neuron, ten stochastic spatiotemporal patterns of synaptic input ("synaptic barrages"; see Materials and Methods) were delivered to progressively more distal $50-\mu \mathrm{m}$ spans of the dendrite. For simulations involving AMPA-only, NMDA-only, or both synaptic conductances, the number of activated synapses within each barrage was iteratively increased at each dendritic location until an action potential was initiated. For each pattern of synaptic input, the threshold number of synapses necessary for action potential generation was determined for inputs occurring at different dendritic locations (incremented at $10-\mu \mathrm{m}$ intervals) and across seven RMPs (-85 to $-55 \mathrm{mV}$ ). As with single EPSPs, identical barrages of synaptic input generated ever-larger local dendritic depolarization at progressively more distal dendritic locations. Whereas the increase in local response amplitude with distance was linear when inputs involved AMPA conductances only, incorporation of the NMDA conductance, by itself or in combination with the AMPA conductance, allowed for supralinear distance-dependent increases in response amplitude and width, including long-lasting regenerative NMDA spikes, at distal dendritic locations. This can best be seen in Figure $2 B$, which shows somatic and local dendritic responses to identical patterns of subthreshold input (determined for each conductance type as the maximal number of synapses that remained subthreshold across all dendritic locations) in a neuron with an RMP of $-70 \mathrm{mV}$.

For AMPA-only synapses, the mean threshold number of synaptic inputs increased with distance from the soma or with hyperpolarization of the RMP (Fig. 2C, left). This result reflects (1) distance-dependent voltage attenuation of summated EPSPs as they transfer to the soma; (2) smaller synaptic currents occurring when driving force is reduced during EPSPs in narrow, high-impedance dendrites (where local EPSP amplitudes are intrinsically larger and bring the membrane potential closer to the synaptic reversal potential); and (3) the necessity for greater depolarization to reach action potential threshold from hyperpolarized RMPs. On the other hand, when synapses contained only the NMDA conductance, the threshold number of synapses decreased with depolarization of the RMP but was fairly uniform across distance in the proximal dendrite before becoming lower at distal locations (Fig. 2C, middle). This shape resulted from distance-dependent voltage attenuation of somatic EPSPs combined 
A

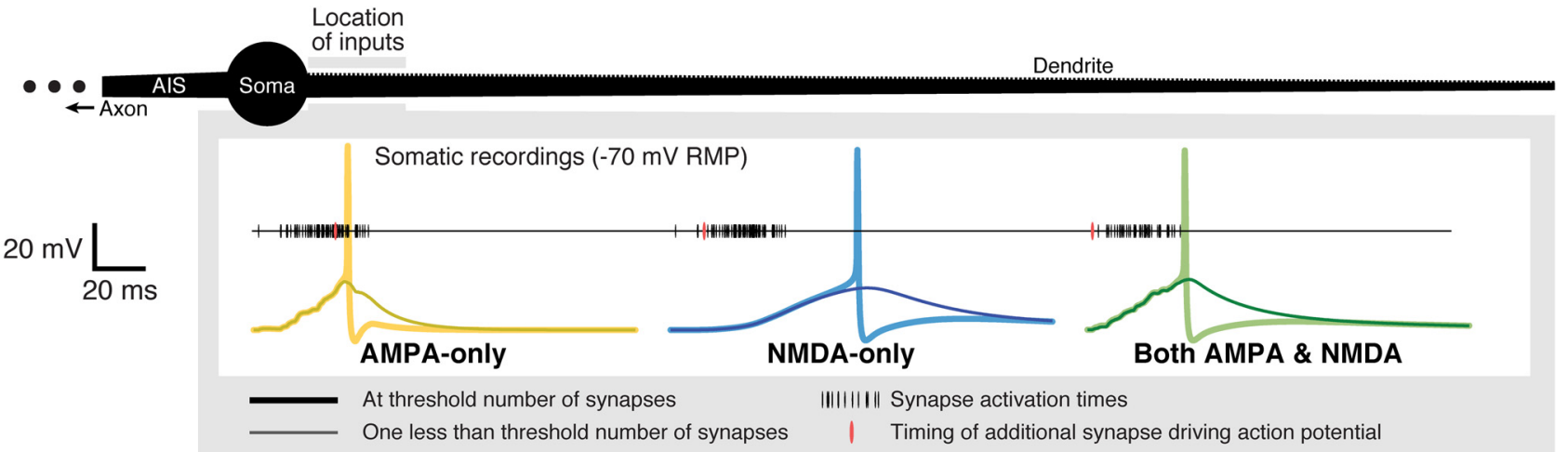

$B_{1}$

$\mathrm{B}_{2}$
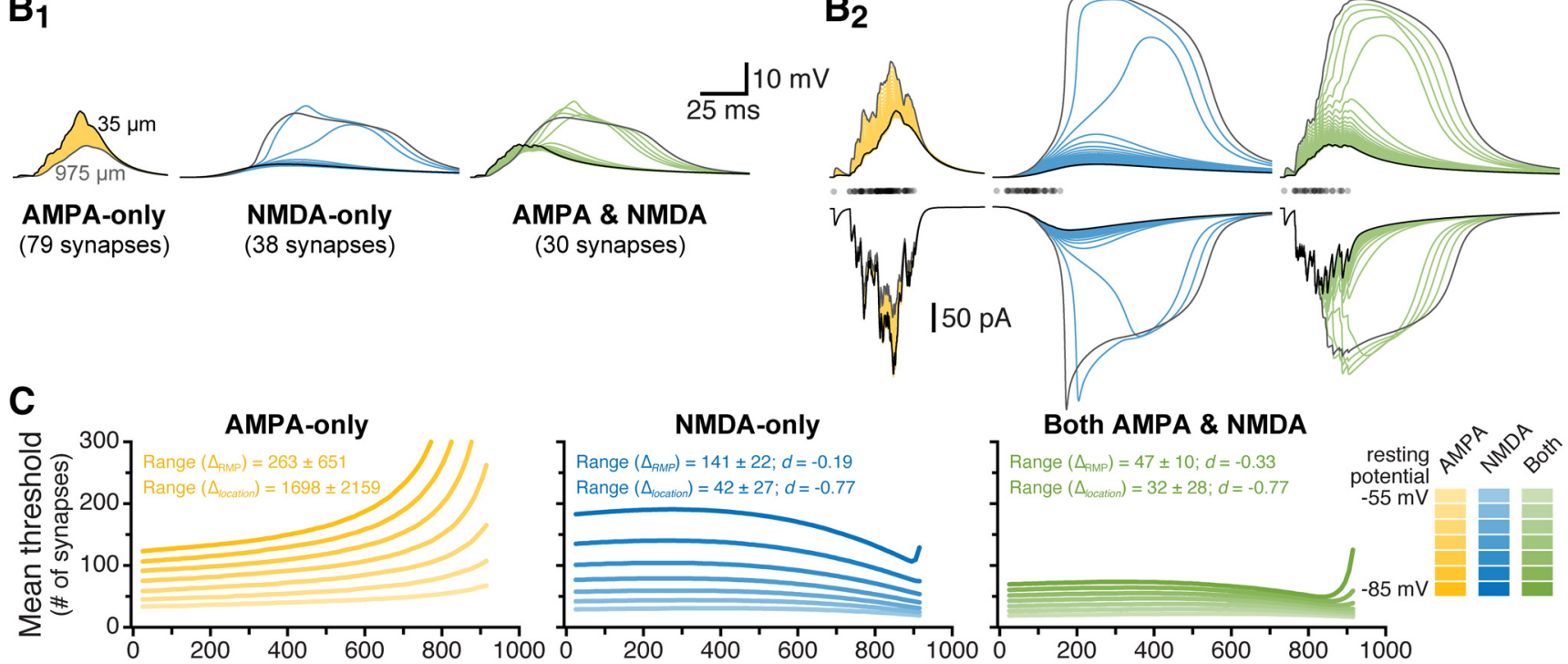

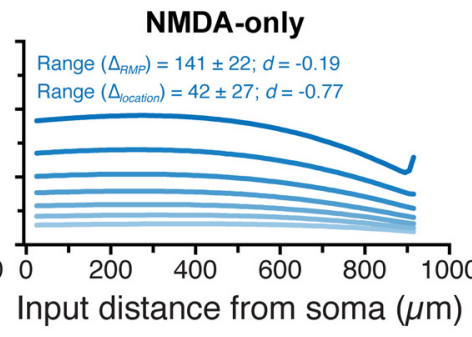

Mean CV across all RMPs (for each dendritic location)

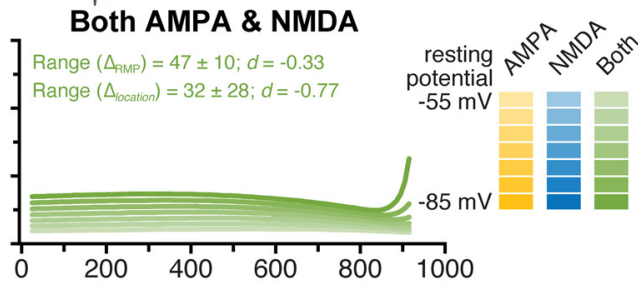

D

Mean CV across all dendritic locations (within each RMP)

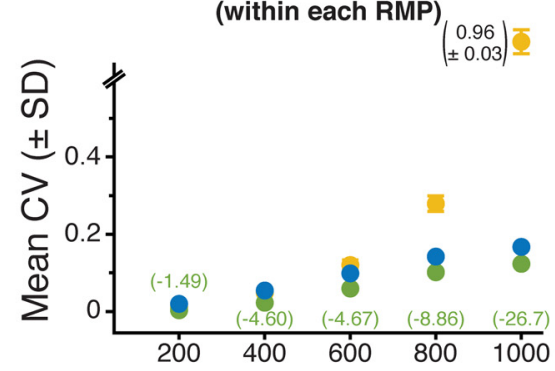

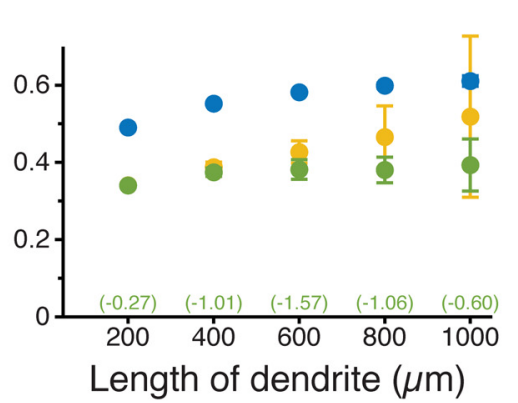

Mean total CV (across all locations and RMPs)

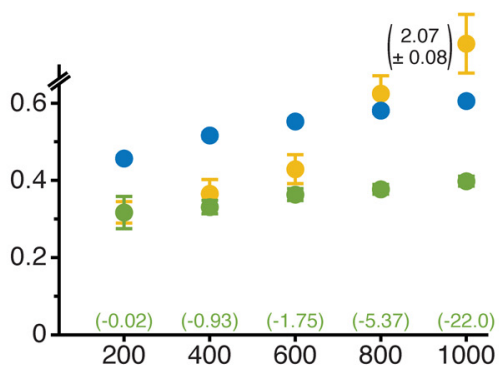

Figure 2. Combining synaptic AMPA and NMDA conductances stabilizes EPSP-spike coupling. $\boldsymbol{A}$, A spiny ball-and-stick neuron (black; $1000-\mu \mathrm{m}$ dendrite) receiving progressively longer iterative trains of a spatiotemporal pattern of synaptic input. Just-threshold (thick traces) and just-subthreshold (thin traces) voltage responses in the soma for AMPA-only (yellow), NMDA-only (blue), or both AMPA and NMDA (green) synaptic conductances in response to inputs arriving within the first $50 \mu \mathrm{m}$ of the dendrite. RMP is $-70 \mathrm{mV}$. $\boldsymbol{B}$, Somatic $\left(\boldsymbol{B}_{1}\right)$ and dendritic $\left(\boldsymbol{B}_{2}\right.$, top) voltage responses for identical subthreshold synaptic barrages at each dendritic location (superimposed; $20-\mu \mathrm{m}$ intervals centered between $35 \mu \mathrm{m}$ [black traces] and $975 \mu \mathrm{m}$ [gray traces] from the soma) for the indicated synaptic conductance types (RMP is $-70 \mathrm{mV}$ ). Summed total synaptic currents are shown in $\boldsymbol{B}_{\mathbf{2}}$, bottom. Timings of synaptic activations are shown with semi-transparent black dots above the synaptic currents in $\boldsymbol{B}_{\mathbf{2}}$. While the spatiotemporal pattern is identical for all synaptic conductances, the number of synapses activated is adjusted for each conductance such that the maximal number of synaptic inputs that remain subthreshold across all dendritic locations is delivered. When occurring at locations where they are "just-subthreshold," somatic EPSPs can recruit somatic and axonal voltage-gated sodium conductances, producing the small peaks visible on the largest somatic EPSPs. $\boldsymbol{C}$, Plots of the mean threshold numbers of synaptic activations necessary to initiate action potentials at different locations in the dendrite for synaptic inputs having AMPA-only (left; yellow), NMDA-only (middle; blue), or 
continued

AMPA and NMDA (right; green) conductances, across seven different RMPs ( -85 to $-55 \mathrm{mV}$ ), as indicated by color depth. Mean ranges of synaptic thresholds measured across RMP $\left(\Delta_{\mathrm{RMP}}\right)$ or dendritic location $\left(\Delta_{\text {location; }} \pm \mathrm{SDs}\right)$ and effect sizes $(d$, in units of $s_{\text {AMPA-only) }}$ of conductance on ranges (for NMDA-only and AMPA+NMDA inputs) are shown in the inset. $\boldsymbol{D}$, Plots of mean CVs ( \pm SDs) calculated for the threshold number of synapses for each pattern of input $(n=10)$, across all locations within each RMPs (left), across all RMPs for each dendritic location (middle), and across all RMPs and dendritic locations (total CV; right) for inputs having AMPA-only (yellow), NMDA-only (blue), or both AMPA and NMDA (green) conductances in dendrites of the indicated lengths. Note that in the 1000- $\mu \mathrm{m}$ dendrite the CV for AMPA-only responses can be very large and off scale. The magnitudes of these large CVs are indicated next to their symbol (SD bars are to scale). The effect sizes $(d)$ for changes in CV with AMPA+NMDA inputs (relative to AMPA-only CVs; expressed in units of $S_{\text {AMPA-only }}$ ) are shown in green for each dendritic length.

with progressively larger synaptic currents at more distal, high-impedance dendritic locations where NMDA spikes of increasing amplitude and duration were observed (see Fig. 2B). Remarkably, when synapses contained both AMPA and NMDA conductances, synaptic thresholds for action potential generation were less sensitive to changes in RMP and less variable across distance than were thresholds for AMPA-only or NMDA-only synaptic inputs (Fig. 2C). While this reduced variability was evident from the mean ranges of synaptic thresholds (Fig. 2C), they were just as robust when threshold variability was normalized as CV. Whether measured across all dendritic locations (within any given RMP; Fig. $2 D$, left), across all
RMPs (for any given dendritic location; Fig. 2D, middle), or across all dendritic locations and RMPs ("total CV"; Fig. 2D, right), and regardless of dendritic length, CVs of synaptic thresholds were always lowest when synapses contained both AMPA and NMDA conductances. Effect sizes were amplified in longer dendrites, likely reflecting their greater electrotonic diversity across input locations (see also Fig. 3).

Total CVs for synaptic thresholds, measured across all dendritic locations and RMPs for AMPA-only trials, ranged from a low of $0.32 \pm 0.03$ (in the $200-\mu \mathrm{m}$ dendrite) to $2.07 \pm 0.08$ (in the $1000-\mu \mathrm{m}$ dendrite; $n=10$ patterns of synaptic input). When synapses incorporated both AMPA
A

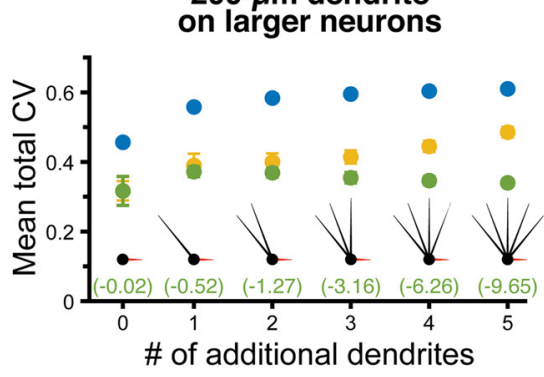

C

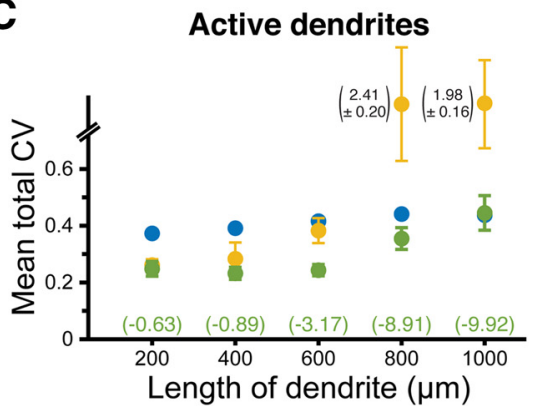

B

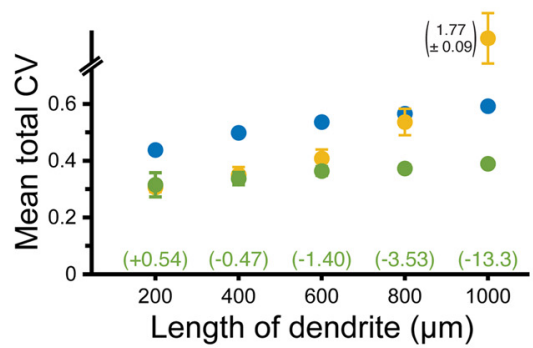

D

Dariable AMPA conductance (600- $\mu \mathrm{m}$ dendrite)

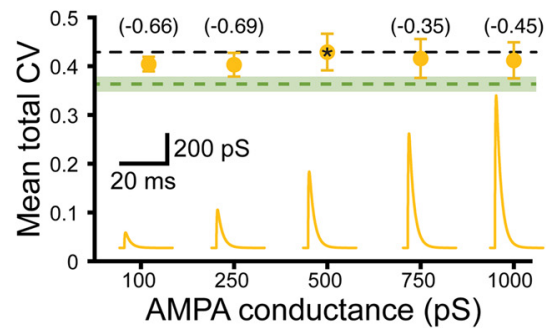

Figure 3. NMDA-dependent stabilization of EPSP-spike coupling is consistent across a range of model conditions and is not dependent on total synaptic conductance. A, Total CVs ( \pm SDs) for synaptic thresholds measured across all dendritic locations and RMPs for AMPA-only (yellow), NMDA-only (blue), and AMPA+NMDA (green) synaptic inputs to a 200- $\mu \mathrm{m}$ dendrite placed on neurons having $0-5$ additional dendrites $(600 \mu \mathrm{m}$ each, as indicated in inset diagrams). Effect sizes for AMPA+NMDA inputs (relative to

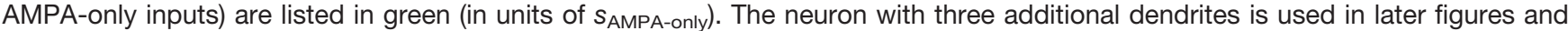
referred to as the "200- $\mu \mathrm{m}$ dendrite on a larger neuron." $\boldsymbol{B}$, Total CVs ( \pm SDs) for synaptic thresholds for AMPA-only (yellow), NMDA-only (blue), and AMPA+NMDA (green) inputs to non-spiny dendrites of the indicated lengths. Effect sizes for AMPA+NMDA CVs shown in green (units of $\left.S_{\text {AMPA-only }}\right)$. C, Total CVs $( \pm S D s)$ for synaptic thresholds measured across all dendritic locations and RMPs for inputs onto spiny dendrites with active conductances (see Table 1). $\boldsymbol{D}$. Plot of mean total CVs for synaptic thresholds $( \pm$ SDs) for AMPA-only EPSPs with the indicated peak conductance magnitudes in a $600-\mu \mathrm{m}$ dendrite. Black dashed line indicates the mean CV of the standard 500 pS AMPA conductance (indicated by asterisk), while the green dashed line indicates mean total CV (with shaded SD) for AMPA (500 pS) + NMDA (1 nS) inputs in the same dendrite. Effect sizes of peak conductance manipulations, relative to the 500 pS AMPA input, are shown in black (in units of $s_{500 p s}$ ). Inset shows the AMPA conductances to scale. 
and NMDA conductances, CVs ranged from $0.32 \pm 0.04$ to $0.40 \pm 0.01$ (in the 200- and 1000- $\mu \mathrm{m}$ dendrites, respectively), with effect sizes (relative to AMPA-only trials) ranging from negligible $\left(-0.02 s_{\text {AMPA-only; }}\right.$ see Materials and Methods) in the 200- $\mu \mathrm{m}$ dendrite, to enormous ( -22 $\left.s_{\text {AMPA-only }}\right)$ in the $1000-\mu \mathrm{m}$ dendrite. Synaptic thresholds for NMDA-only conductances were always more variable than for inputs having both conductances, with total CVs ranging from $0.46 \pm 0.01$ to $0.61 \pm 0.01$ (in dendrites of 200 and $1000 \mu \mathrm{m}$, respectively) and effect sizes ranging

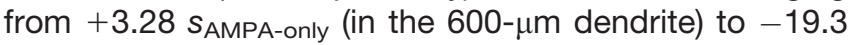

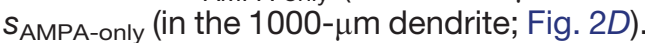

To test whether the impact of dendritic length on synaptic threshold variability was reflective of electrotonic structure of the neuron, we measured synaptic thresholds in a $200-\mu \mathrm{m}$ dendrite attached to larger neurons having one to five additional dendrites (each of $600 \mu \mathrm{m}$; Fig. 3A). Even as the measured dendrite remained static (at $200 \mu \mathrm{m}$ ), the effect size of combining AMPA and NMDA conductances on synaptic threshold variability was enhanced as neurons became progressively larger, ranging from almost nothing $\left(-0.02 s_{\text {AMPA-only }}\right)$ in the smallest neuron having no additional dendrites, to very large $\left(-9.65 s_{\text {AMPA-only }}\right)$ in the largest neuron with five additional dendrites (Fig. $3 A$ ). Similarly, making our normal ball-andstick neurons electrotonically "tighter" by removing dendritic spines moderately reduced the impact of synaptic conductance on threshold variability (Fig. 3B; compare with the total CVs in Fig. 2D).

To test whether the impact of NMDA conductance depends on passive dendrites, in some simulations we added dendritic voltage-gated sodium and potassium conductances (see Table 1). Combining AMPA and NMDA conductances continued to reduce the variability of synaptic thresholds relative to AMPA-only inputs when dendrites contained active conductances (Fig. $3 C$ ).

Finally, to test whether the reduced variability in synaptic thresholds for AMPA+NMDA inputs was because of their larger peak conductance magnitudes, we repeated simulations using a range of AMPA-only conductances (100 pS to $1 \mathrm{nS}$ ) in a $600-\mu \mathrm{m}$ dendrite. We found no consistent relationship between peak AMPA conductance and total CV of EPSP-spike coupling measured across all dendritic locations and RMPs (Fig. 3D; see also Fig. 7). This suggests that, rather than reflecting larger total synaptic conductance, the enhanced fidelity of EPSP-spike coupling observed with AMPA+NMDA inputs depends instead on properties intrinsic to the NMDA conductance.

\section{Synergistic interaction of voltage dependence and slow kinetics of the NMDA conductance}

The NMDA conductance differs from the AMPA conductance in two key ways: it is voltage dependent and has slower kinetics. To determine the relative impact of these properties on EPSP-spike coupling, we modified our simulations to measure variability in synaptic thresholds independently across location and RMP (Fig. 4). To do this, we started with our AMPA-only model (500 pS) and then replaced a portion of that conductance (100 or 250 pS) with either (1) the normal NMDA conductance; (2) a conductance having NMDA-like voltage dependence with AMPA-like kinetics ("fast-NMDA"); or (3) a conductance with NMDA-like kinetics but lacking voltage dependence ("slow-AMPA"). These added conductances were titrated so that each model exhibited a similar synaptic threshold for inputs to the proximal end of the 600- $\mu \mathrm{m}$ dendrite at $-70 \mathrm{mV}$ ( $\sim 51$ synapses). The resulting synaptic conductances are shown to scale in Figure $4 A$ following activation of individual synapses under voltage-clamp (voltage-dependent conductances shown for activations at -85 and $-55 \mathrm{mV}$, as indicated). As in Figure 2, for each dendritic location $(50-\mu \mathrm{m}$ spans, incremented at $10-\mu \mathrm{m}$ intervals), and RMP (from -85 to $-55 \mathrm{mV}$, in $5-\mathrm{mV}$ increments), we measured synaptic thresholds for action potential generation in response to each of ten randomized spatiotemporal patterns of synaptic input (Fig. 4B). We then calculated the $\mathrm{CV}$ of synaptic thresholds across RMPs for each dendritic location (Fig. 4C), across all dendritic locations at a given RMP (Fig. 4D), and across all dendritic locations and RMPs (total CV; Fig. 4E). This allowed us to dissociate the relative impacts of voltage dependance and conductance kinetics on the fidelity of EPSP-spike coupling. Adding back an AMPA conductance with slower kinetics (the brown plots from simulations including the slow-AMPA conductance in Fig. 4C) modestly reduced the $\mathrm{CV}$ of synaptic thresholds measured across RMP at a given dendritic location, especially in shorter dendrites or in proximal locations in longer dendrites. On the other hand, adding NMDA-like voltage dependence with fast, AMPA-like, kinetics (fast-NMDA; purple plots in Fig. $4 C$ ) increased the CV of synaptic thresholds measured across RMP at individual dendritic locations in small and/or proximal dendrites. Both of these effects were "dose dependent," in that they were larger when they contributed proportionally more to the total synaptic conductance (Fig. 4C, compare dashed vs solid lines).

When our normal NMDA conductance (titrated, as above) was added to these smaller AMPA conductances (Fig. 4C, green traces), CVs for synaptic thresholds measured across RMP were intermediate to those generated by fast-NMDA and slow-AMPA conductances, being only marginally larger than for AMPA-only inputs in short dendrites (or at proximal dendritic locations in longer dendrites) but becoming progressively lower at more distal dendritic locations. Overall, mean CVs of synaptic thresholds measured across RMP at all dendritic locations with titrated NMDA conductances were lower than for AMPAonly inputs in all dendrites longer than $200 \mu \mathrm{m}$, and in the $200-\mu \mathrm{m}$ dendrite when attached to a larger neuron with three additional $600-\mu \mathrm{m}$ dendrites (effect sizes ranging from $-0.26 s_{\text {AMPA-only }}$ in the $400-\mu \mathrm{m}$ dendrite to -1.91 $S_{\text {AMPA-only }}$ in the $200-\mu \mathrm{m}$ dendrite attached to a larger neuron). Effect sizes of conductance-dependent changes in synaptic threshold variability across RMP are plotted for each dendritic location in Figure $5 \mathrm{~A}$.

The impact of synaptic conductance on synaptic threshold variability was quite different when measured across all dendritic locations within a given RMP (Fig. 4D). For all dendrites $>200 \mu \mathrm{m}$ (and including in the $200-\mu \mathrm{m}$ 

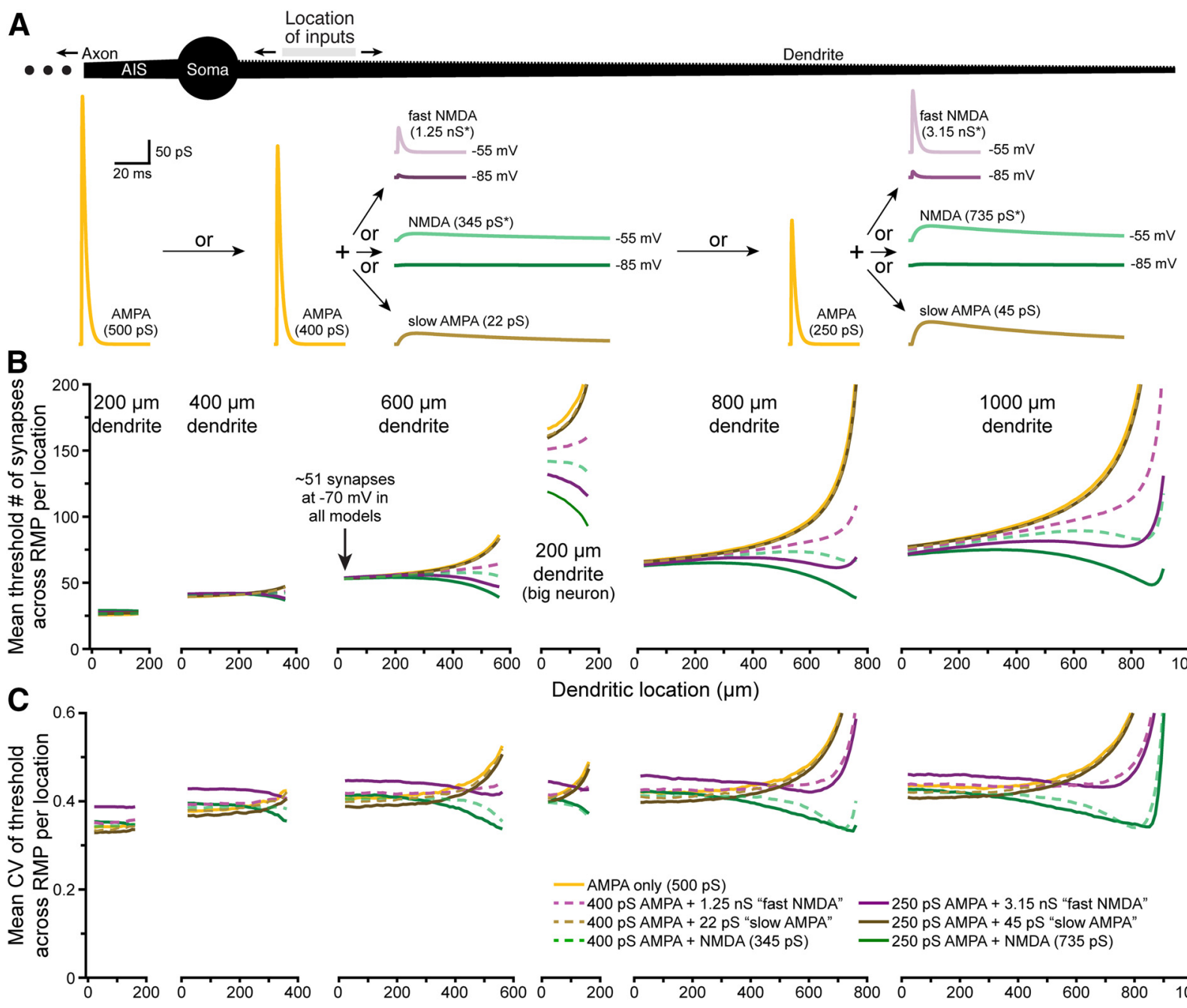

Dendritic location $(\mu \mathrm{m})$
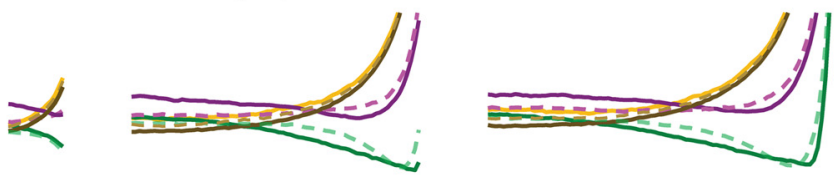

AMPA only (500 pS)

- - 400 pS AMPA + 1.25 nS "fast NMDA" - 250 pS AMPA + 3.15 nS "fast NMDA"

- - 400 pS AMPA + 22 pS "slow AMPA" - 250 pS AMPA + 45 pS "slow AMPA"

- -400 pS AMPA + NMDA (345 pS) -250 pS AMPA + NMDA (735 pS)
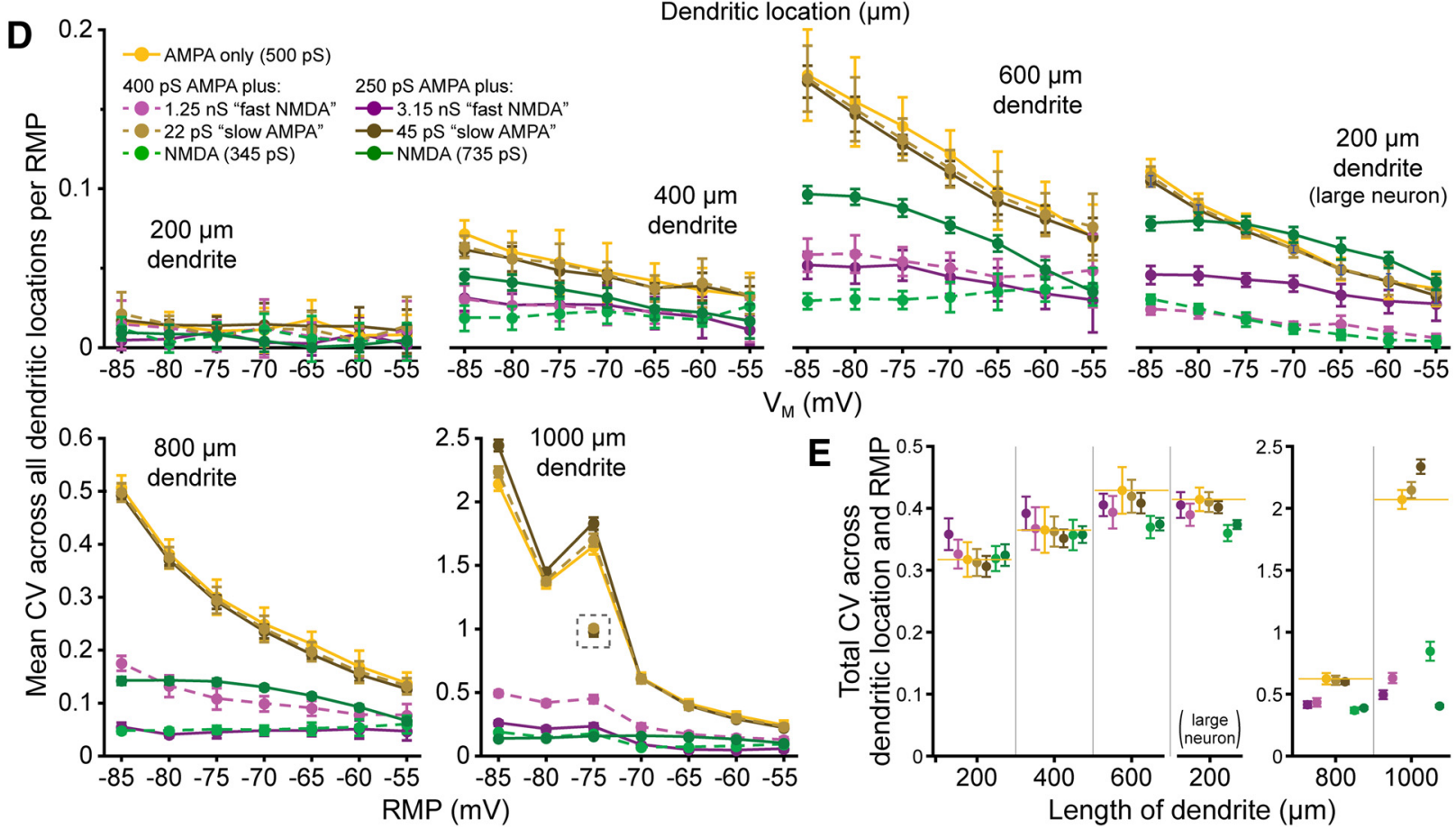
continued

Figure 4. Differential impact of voltage dependence and kinetics on the fidelity of synaptic integration. $\boldsymbol{A}$, Diagram of a ball-andstick neuron (top) receiving spatiotemporal patterns of synaptic input to $50-\mu \mathrm{m}$ spans of dendrite to determine the number of synaptic inputs necessary for action potential generation. Traces below show the synaptic conductances tested: a 500 pS AMPA-only conductance, a 400 pS AMPA conductance "doped" with one of three additional conductances, including a fast-NMDA conductance ("nominally $1.25 \mathrm{nS}$ maximal conductance, reaching $4.6 \mathrm{pS}$ at $-85 \mathrm{mV}$, and $49 \mathrm{pS}$ at $-55 \mathrm{mV}$, as shown), a slow-AMPA conductance with NMDA kinetics (22 pS), or a nominally 345 pS NMDA conductance (*reaching 1.3 and $13.6 \mathrm{pS}$ at -85 and $-55 \mathrm{mV}$, respectively), or a 250 pS AMPA conductance doped with relatively larger fast-NMDA, slow-AMPA, and NMDA conductances). All conductances are shown to scale and were titrated such that each generated a mean threshold of $\sim 51$ synapses in the proximal end of the $600-\mu \mathrm{m}$ dendrite at $-70 \mathrm{mV}$ (arrow in $\boldsymbol{B}$ ). $\boldsymbol{B}$, Plots of mean synaptic thresholds for inputs across all RMPs for each $50-\mu \mathrm{m}$ span of dendrite (incremented at 10- $\mu \mathrm{m}$ intervals) for inputs having the indicated synaptic conductances in dendrites of the indicated lengths ( $y$-axis cut off at 200 synapses; color coding as in panel $\boldsymbol{C}$ ). Note that we also tested a $200-\mu \mathrm{m}$ dendrite attached to a large neuron having three additional $600-\mu \mathrm{m}$ dendrites (middle plot; see also Fig. $3 A$ ). C, Plots of mean CVs for thresholds calculated across seven RMPs $(-85$ to $-55 \mathrm{mV}$ ) for each color-coded synaptic conductance at each dendritic location (i.e., measures of RMP-dependent variability of threshold for each given dendritic location) for the different length dendrites (as indicated in $\boldsymbol{B}$ ); $y$-axis cut off at 0.6. D. Plots of mean CVs $( \pm S D s)$ for synaptic thresholds for each color-coded conductance measured across all dendritic locations within each RMP (i.e., a measure of location-dependent variability in threshold for each RMP). For trials in the 1000- $\mu$ m dendrite at $-75 \mathrm{mV}$, the $500 \mathrm{pS}$ AMPA and slow AMPA models hit very high thresholds $(>3000)$ in their most distal measurable compartment, which generated large CVs at that RMP. CVs calculated for those conductances without the last dendritic location are plotted in the gray dashed box. $\boldsymbol{E}$, Plots of mean total CVs ( \pm SDs) calculated across all dendritic locations and all RMPs for neurons with the indicated dendritic lengths and color-coded synaptic conductances.

dendrite when attached to a larger neuron), the presence of the fast-NMDA conductance greatly lowered the CV of synaptic thresholds within any given RMP, while adding the slow-AMPA conductance had little impact on synaptic threshold variability across dendritic locations. The combination of voltage dependence and slow kinetics (i.e., our titrated standard NMDA conductance; green traces) generally lowed CV for synaptic thresholds measured across dendritic locations for a given RMP. For both fastNMDA and regular NMDA conductances, the dose dependency was nonlinear, as the smaller dose typically generated the largest reductions in CV (relative to AMPAonly conductance; Fig. 4D). The effect sizes of these synaptic conductances on threshold variability across location within a given RMP (relative to 500 pS AMPA-only inputs) are shown in Figure 5B.

Finally, we measured total CV of synaptic thresholds across all dendritic locations and all RMPs for ten patterns of synaptic input (Fig. 4E). In smaller neurons (those with 200- and 400- $\mu \mathrm{m}$ dendrites), adding the fast-NMDA conductance (purple symbols) slightly increased CV relative to AMPA conductance alone. On the other hand, adding slow-AMPA kinetics (brown symbols) modestly reduced $\mathrm{CV}$ relative to AMPA-only inputs in most models. These effects were reversed in larger neurons such that the slow-AMPA conductance increased mean total CV in the $1000-\mu \mathrm{m}$ dendrite, while the voltage-dependent fastNMDA conductance reduced total $\mathrm{CV}$ for all dendrites longer than $400 \mu \mathrm{m}$, as well as in the $200-\mu \mathrm{m}$ dendrite when it was attached to a larger neuron. This likely reflects the ability of the fast-NMDA conductance to effectively lower CV across distance in larger neurons by avoiding or delaying the exponential rise in synaptic thresholds observed for AMPA-only inputs (Fig. 4C), and that slower kinetics (i.e., slow-AMPA models) have reduced efficacy in lowering CV across RMPs at distal dendritic locations (Fig. 4C).

Combining voltage dependence with slow kinetics (i.e., the titrated normal NMDA conductance; Fig. 4E, green symbols) led to overall lower total CVs for synaptic thresholds in all dendrites longer than $200 \mu \mathrm{m}$, including in the $200-\mu \mathrm{m}$ dendrite when attached to a larger neuron. Mean total CVs (across 10 synaptic input patterns) and effect sizes for conductance-dependent changes in total CV (relative to the 500 pS AMPA-only inputs) are shown in Table 2. In most dendrites, combining voltage dependence with slower kinetics had a synergistic effect in lowering total CV of synaptic thresholds to a greater extent than either manipulation on its own, often with "large" effect sizes (i.e., $>1 s_{\text {AMPA-only }}$ ).

To further compare the impact of synaptic conductance on the variability of EPSP-spike coupling, as measured across all RMPs (for a given dendritic location) or across all dendritic locations (for a given RMP), we plotted the effect sizes for our various synaptic conductances (relative to threshold variability observed for AMPA-only inputs) in Figure 5. This essentially transforms data in Figure $4 C, D$ into measures of effect size (in units of $s_{\text {AMPA-only) }}$ ) to allow comparison of the differential impact of voltage dependence and slow kinetics on synaptic threshold variability across RMPs (Fig. $5 A$ ) or across all dendritic locations (Fig. 5B). Included in Figure 5 are results from our normal AMPA+NMDA conductance (500 pS AMPA + 1 nS NMDA; thick semi-transparent gray lines), which tended to track closely to the titrated NMDA conductances (green lines). As shown in Figure $5 A$, adding the fastNMDA conductance induced a dose-dependent increase in the CVs for synaptic thresholds across RMPs in shorter dendrites and in the proximal two-thirds of longer dendrites (those $\geq 600 \mu \mathrm{m}$ ), with effect sizes for the larger dose of fast-NMDA (3.15 nS, paired with 250 pS AMPA) averaging $+1.08 \pm 0.26 s_{\text {AMPA-only (i.e., more variable }}$ thresholds across RMP relative to AMPA-only inputs) in shorter $(200$ and $400 \mu \mathrm{m})$ dendrite models and in the proximal two-thirds of longer dendrites. At distal locations in longer dendrites, effect sizes for NMDA-containing models became negative (i.e., reduced variability across RMP) and very large relative to AMPA-only inputs (Fig. 5). This 
A

Effect size of synaptic conductance on CV measured across RMP at each dendritic location

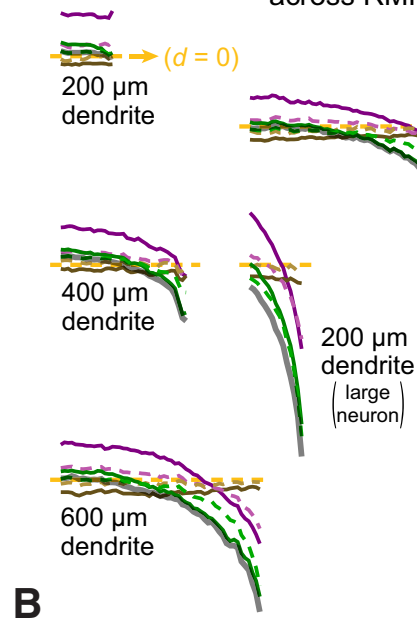
dendrite
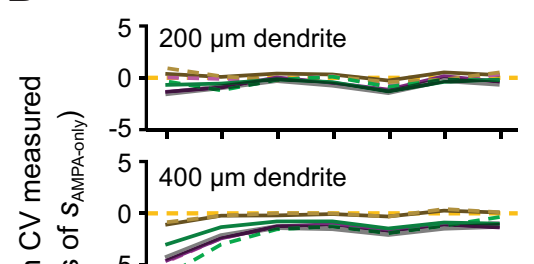

$=-d=0$ (as AMPA-only, $500 \mathrm{pS}$ )

$=400$ pS AMPA + 1.25 nS "fast NMDA" -250 pS AMPA + 3.15 nS "fast NMDA" $=-400$ pS AMPA + 22 pS "slow AMPA" $=-400$ pS AMPA + 22 pS "slow AMPA -250 pS AMPA + 46 pS "slow AMPA"
$-=400$ pS AMPA + NMDA (345 pS) $250 \mathrm{pS}$ AMPA + NMDA (735 pS) 500 pS AMPA + 1 nS NMDA
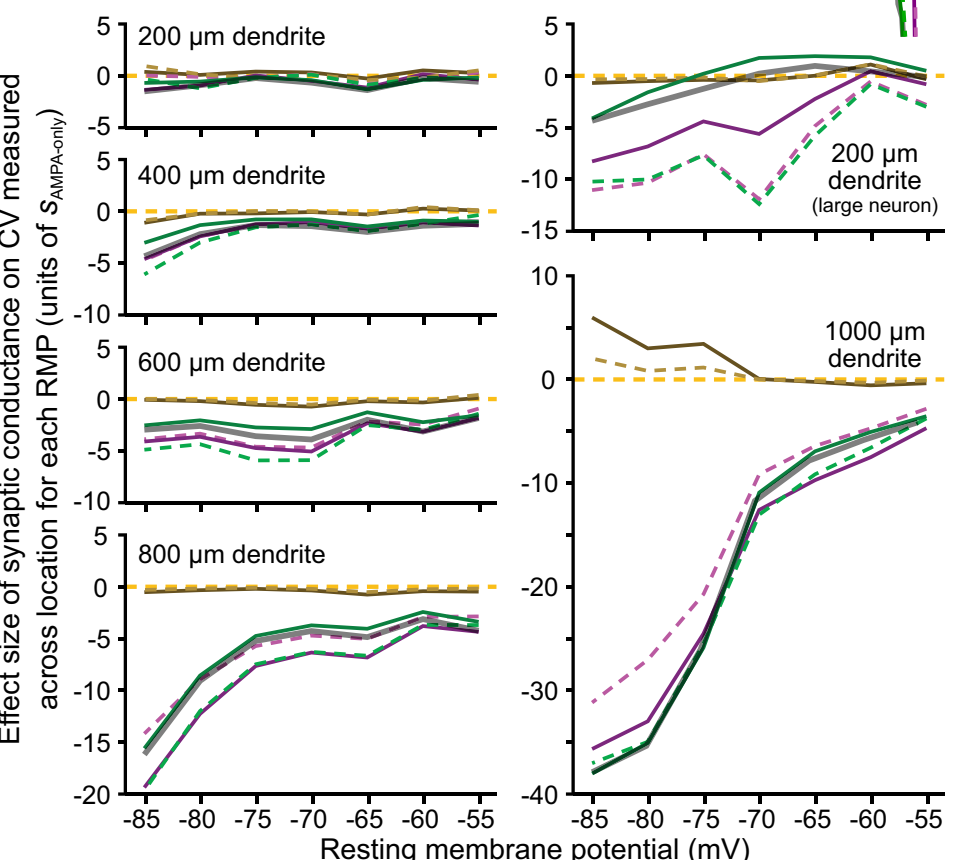

Figure 5. Effect sizes of synaptic conductance on variability of EPSP-spike coupling across RMP and dendritic location. $\boldsymbol{A}$, Plots of effect sizes of conductance-dependent changes in CV calculated across RMP for each dendritic location (in units of $s_{\text {AMPA-only; }}$ see Materials and Methods). Yellow dashed lines indicate $d=0$ (i.e., identical to $500 \mathrm{pS}$ AMPA-only trials), with effect sizes above that line reflecting larger-than-AMPA-only CVs, while values below the dashed-yellow lines indicate smaller-than-AMPA-only CVs. Data are from Figure $4 C$, and conductance types are similarly indicated by color. Effect sizes of our normal AMPA (500 pS) conductance combined with 1 nS NMDA (i.e., from Fig. 2C) are also shown with thick gray semi-transparent lines. Inputs with the fast-NMDA conductance tended to generate more variability in synaptic thresholds across RMP, especially in short or proximal dendrites, while inputs with slow kinetics exhibited smaller CVs relative to AMPA-only inputs. Combining voltage dependence with slow kinetics (e.g., green and gray NMDA traces) reduced variability in synaptic thresholds across RMP at all locations in most dendrites. Plots for the

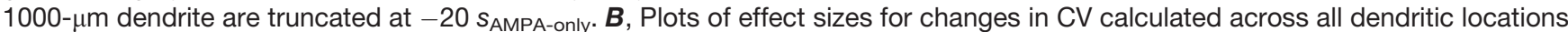
within each RMP for data shown in Figure $4 D$ (conductance types indicated by color, as in A). Voltage-dependent conductances greatly reduced threshold variability across location within a given RMP (an effect magnified at hyperpolarized RMPs), whereas slow kinetics in the absence of voltage dependence had little effect.

occurred because adding a voltage-dependent component (regardless of kinetics) avoided, or at least delayed, the distance-dependent exponential climb in synaptic thresholds exhibited by AMPA-only inputs at distal dendritic locations (see Fig. $4 B$ ) and the corresponding increases in synaptic threshold variability across RMPs (see Fig. 4C). On the other hand, adding the larger dose of slow-AMPA conductance moderately lowered the variability of synaptic thresholds across RMPs for most proximal dendritic locations, with effect sizes averaging $-0.46 \pm 0.13 s_{\text {AMPA-only in all }}$ models $>200 \mu \mathrm{m}$, including in the $200-\mu \mathrm{m}$ dendrite when attached to a larger neuron (Fig. 5A). 
Table 2: Effect sizes for Figure 4E

\begin{tabular}{|c|c|c|c|c|c|c|c|c|c|}
\hline Dendritic length $(\mu \mathrm{m})$ & Conductance & gAMPA & $\begin{array}{l}\text { Mean } \\
\text { "total" CV }\end{array}$ & SD & $\begin{array}{l}\text { Effect } \\
\text { size }(d)\end{array}$ & gAMPA & $\begin{array}{l}\text { Mean } \\
\text { "total" CV }\end{array}$ & SD & $\begin{array}{l}\text { Effect } \\
\text { size }(d)\end{array}$ \\
\hline \multirow{2}{*}{200} & AMPA + fast NMDA & $400 \bigcirc$ & 0.326 & 0.023 & 0.325 & 250 & 0.358 & 0.025 & 1.46 \\
\hline & AMPA+NMDA & $400 \bigcirc$ & 0.319 & 0.020 & 0.057 & 250 & 0.325 & 0.017 & 0.263 \\
\hline \multirow[t]{2}{*}{400} & AMPA-only & 500 & 0.365 & 0.280 & & & & & \\
\hline & AMPA + fast NMDA & 400 & 0.367 & 0.034 & 0.060 & 250 & 0.392 & 0.027 & 0.721 \\
\hline \multirow[t]{4}{*}{600} & AMPA-only & 500 & 0.429 & 0.038 & & & & & \\
\hline & AMPA + fast NMDA & $400 \bigcirc$ & 0.394 & 0.026 & -0.940 & 250 & 0.406 & 0.018 & -0.623 \\
\hline & AMPA + slow AMPA & $400 \bigcirc$ & 0.420 & 0.027 & -0.253 & 250 & 0.409 & 0.016 & -0.546 \\
\hline & $\mathrm{AMPA}+\mathrm{NMDA}$ & 400 & 0.370 & 0.018 & -1.578 & 250 & 0.375 & 0.010 & -1.450 \\
\hline \multirow[t]{2}{*}{200 (on large neuron) } & AMPA-only $\bigcirc$ & 500 & 0.414 & 0.019 & & & & & \\
\hline & AMPA + fast NMDA & $400 \bigcirc$ & 0.390 & 0.018 & -1.308 & $250 \bullet$ & 0.405 & 0.021 & -0.476 \\
\hline \multirow{2}{*}{800} & AMPA + slow AMPA & 400 & 0.612 & 0.037 & -0.283 & 250 & 0.601 & 0.020 & -0.520 \\
\hline & AMPA+NMDA & $400 \bigcirc$ & 0.369 & 0.023 & -5.537 & 250 & 0.389 & 0.010 & -5.104 \\
\hline \multirow[t]{4}{*}{1000} & AMPA-only & 500 & 2.071 & 0.076 & & & & & \\
\hline & AMPA + fast NMDA & $400 \bigcirc$ & 0.630 & 0.041 & -18.964 & 250 & 0.495 & 0.036 & -20.737 \\
\hline & AMPA + slow AMPA & $400 \bigcirc$ & 2.147 & 0.065 & 1.002 & 250 & 2.337 & 0.058 & 3.493 \\
\hline & AMPA+NMDA & $400 \bigcirc$ & 0.847 & 0.077 & -16.111 & 250 & 0.405 & 0.010 & -21.928 \\
\hline
\end{tabular}

Listed are the mean "total" CVs, SDs, and effect sizes (relative to AMPA-only synapses) for synaptic thresholds calculated across all dendritic locations and all RMPs averaged from 10 patterns of synaptic input for each type of synaptic conductance (color coded to data in Fig. 4E). Effect sizes (d) indicate the normalized mean difference in CV relative to the CV for the 500 pS AMPA-only conductance, in units of sAMPA-only (see Materials and Methods). Sign indicates the direction of change [larger $(+)$ or smaller $(-)$ mean CVs than AMPA-only inputs]. Light shading (blue $=$ smaller CVs, red $=$ larger CVs) identifies manipulations generating mean changes in CV $>0.5 \mathrm{SD}$, while darker shadings indicate mean changes $>1.0 \mathrm{SD}$ of the AMPA-only result. Note that AMPA+NMDA conductances generated consistently larger reductions in CV relative to manipulations that provided voltage dependence or slower kinetics alone. The large neuron with a 200 - $\mu$ m dendrite (tested) included three additional 600- $\mu$ m dendrites (as in Fig. $3 A$ ).

Combining voltage dependence with slow kinetics (Figs. $4 C, 5 A$, the green "NMDA" traces) revealed a location-dependent reduction in variability of synaptic thresholds measured across RMPs that was similar to that observed when adding the fast-NMDA conductance (Fig. 5A, compare green and purple traces). However, relative to fast NMDA trials, the slower kinetics of the normal NMDA conductance consistently led to lower CVs for synaptic thresholds at all dendritic locations in all model neurons. Mean effect sizes for all dendritic locations ranged from negligible $(+0.06$ $s_{\text {AMPA-only }}$ in the $200-\mu \mathrm{m}$ dendrite) to very large $(-5.2$ in the $1000-\mu \mathrm{m}$ dendrite), and always became larger with increasing distance from the soma. As with the fast-NMDA conductance, the enormous effect sizes in distal dendrites reflects primarily the earlier (i.e., at more proximal dendritic locations) exponential increases in synaptic thresholds observed in AMPA-only models, rather than the more modest distance-dependent reduction in CV occurring in the presence of the NMDA conductance (Fig. 4C).

The impact of synaptic conductance on synaptic threshold variability measured across location within a given RMP is shown in Figure 5B. Regardless of kinetics, the addition of voltage dependence greatly reduced the CV of synaptic thresholds across location (Fig. 5B), and this effect was magnified in larger dendrites and at more hyperpolarized RMPs. The presence of slow kinetics in the absence of voltage dependence had little impact on synaptic threshold variability across dendritic location, except in the longest dendrite $(1000 \mu \mathrm{m})$ at hyperpolarized RMPs, where variability increased relative to AMPA-only inputs.

Together, these results indicate that the impact of the NMDA conductance on the variability of synaptic thresholds across dendritic location and RMP reflects a synergistic interaction of multiple properties of the NMDA conductance, with its voltage dependence primarily enhancing fidelity across dendritic location within any given RMP, and its slower kinetics tending to enhance fidelity across RMP at a given dendritic location. These properties act together to greatly reduce the variability of EPSP-spike coupling across both RMP and dendritic location, relative to AMPA-only synaptic inputs.

\section{Synaptic integration in morphologically realistic neurons}

The simulations above reveal that, in simplified neuron morphologies, the presence of both AMPA and NMDA conductances at synapses reduces location-dependent and RMP-dependent variability of somatic responses to spatially restricted barrages of synaptic input. To determine the impact of synaptic conductance on synaptic integration in realistic neuronal morphologies, we placed spinous synapses at $1-\mu \mathrm{m}$ intervals across the dendritic trees of two reconstructed neurons: a relatively large CA3 

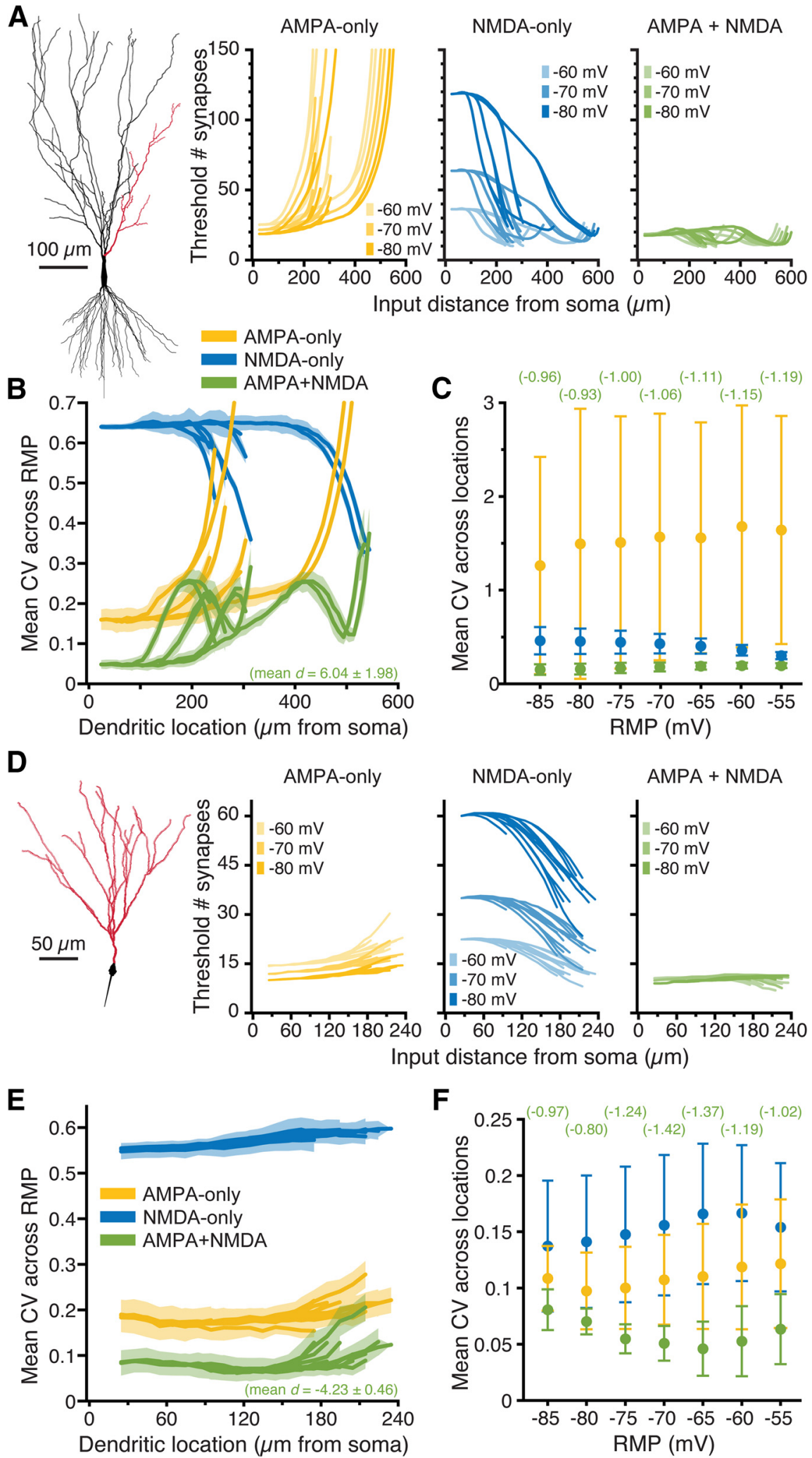

Figure 6. Impact of synaptic conductance on synaptic integration in realistic neuron morphologies. $\boldsymbol{A}$, Plots of the mean threshold number of synaptic activations necessary to depolarize the soma of a CA3 pyramidal neuron by $3 \mathrm{mV}$ at progressively more distal $50-\mu \mathrm{m}$ dendritic segments (along red dendrites in diagram at left) experiencing ten static-random spatiotemporal patterns of synaptic input. Colors (yellow, blue, and green) indicate simulations with AMPA, NMDA, and both AMPA and NMDA conductances, respectively, at three different RMPs $(-60,-70$, and $-80 \mathrm{mV}) ; y$-axes are truncated at 150 synapses to show differences in proximal dendrites. $\boldsymbol{B}$, Plots of mean CVs for synaptic thresholds calculated across seven RMPs ( -85 to $-55 \mathrm{mV}$ ) for each dendritic location. 
continued

Synaptic conductances indicated by color, with shaded regions indicating SDs. The mean effect size ( $d$, in units of $\left.s_{\text {AMPA-only }}\right)$ for CVs from AMPA-plus-NMDA simulations (relative to AMPA-only conductance) is shown in green at the bottom of the graph. $\boldsymbol{C}$, Comparisons of mean ( \pm SD) CVs calculated for synaptic thresholds across dendritic locations within each of seven RMPs. Colors as in $\boldsymbol{B}$. Effect sizes for AMPA-plus-NMDA trials (vs AMPA-only inputs) are shown in green at top for each RMP (units of $\boldsymbol{S}_{\text {AMPA-only). }}$ $\boldsymbol{D}$, Plots of the threshold number of synaptic activations necessary to drive a 5-mV somatic depolarization in a dentate granule neuron (red dendrites in diagram to left) at progressively more distal 50- $\mu \mathrm{m}$ segments experiencing ten expanding static-random spatiotemporal patterns of synaptic input. Colors as in $\boldsymbol{B}$. $\boldsymbol{E}$, Plots of mean CVs (with SD in shaded regions) of synaptic thresholds measured across seven RMPs for each dendritic location in the dentate granule neuron. Synaptic conductances indicated by color. $\boldsymbol{F}$, Comparisons of mean CV $( \pm S D)$ for synaptic thresholds calculated across dendritic location within each of seven RMPs in the granule neuron. Effect sizes of AMPA-plus-NMDA inputs (relative to AMPA-only inputs) are indicated in green at top for each RMP (units of $S_{\text {AMPA-only). }}$.

pyramidal neuron (Fig. $6 A$ ), and a smaller dentate granule neuron (Fig. 6D). Because of their large dendritic arbors, it was not possible to drive action potential generation in these neurons with spatially restricted $(50-\mu \mathrm{m})$ synaptic barrages, as even extreme depolarization of distal dendrites (e.g., clamping $50-\mu \mathrm{m}$ spans to $0 \mathrm{mV}$ ) failed to depolarize the AIS to action potential threshold. Instead, we set arbitrary thresholds of somatic depolarization $(3 \mathrm{mV}$ above RMP for the CA3 neuron, and $5 \mathrm{mV}$ above RMP for the smaller dentate granule neuron) and iteratively activated expanding stochastic patterns of synaptic input at different dendritic locations until somatic voltage thresholds were realized. RMPs were nominally set between -85 and $-55 \mathrm{mV}$, in $5-\mathrm{mV}$ increments, and synaptic barrages delivered to $50-\mu \mathrm{m}$ spans along the dendritic branches indicated in red in Figures $6 A, D$ (at 10- $\mu \mathrm{m}$ increments). In the CA3 neuron, the threshold number of AMPA-only inputs necessary to generate a 3-mV somatic depolarization increased with distance from the soma, or with depolarization of the RMP, as voltage attenuation and reduced driving force impaired synaptic depolarization of the soma (Fig. $6 A$, yellow plots). In contrast, when synapses contained only the NMDA conductance, the threshold number of synapses to depolarize the soma by $3 \mathrm{mV}$ decreased with distance from the soma and/or with depolarization of the RMP, as distance-dependent increases in local input impedance, and/or depolarization of the RMP, enhanced the voltage-dependent NMDA conductance (Fig. 6A, blue plots). Combining both AMPA and NMDA conductances generated threshold numbers of synapses that were generally lower and more uniform across dendritic location and RMP (Fig. 6A, green plots). Whether measured across RMP (for a given dendritic location; Fig. $6 B$ ) or across dendritic location (within a given RMP; Fig. 6C), CVs were always less when AMPA and NMDA conductances were combined. Across RMPs, CVs of AMPA+NMDA inputs were reduced, on average, by $6.04 \pm 1.98 s_{\text {AMPA-only }}$ (Fig. $6 B$ ), while effect sizes for reductions in $\mathrm{CV}$ across dendritic location (within a given RMP) ranged from -0.93 to -1.19 , primarily because of large variability in the CVs of AMPA-only trials (Fig. $6 C$, compare the SDs of AMPA-only and AMPA+NMDA trials).

Across all dendritic locations and RMPs, total CVs in AMPA-only trials averaged $1.83 \pm 0.10$ across ten patterns of synaptic input, but were only $0.19 \pm 0.01$ in AMPA+NMDA trials, with an effect size of -16.6 $S_{\text {AMPA-only }}$ for AMPA+NMDA inputs relative to AMPA- only trials. These results demonstrate that the combined presence of synaptic AMPA and NMDA conductances greatly reduces distance-dependent and RMPdependent variability of synaptic integration (relative to AMPA-only inputs) in a realistic neuron morphology.

Similar results were observed in the smaller dentate granule neuron (Fig. $6 D$ ), where the threshold number of AMPA-mediated synaptic inputs increased with distance from the soma, or with depolarization of the RMP (Fig. $6 D$, yellow plots), because of distance-dependent voltage attenuation and reduced synaptic driving force, respectively. Conversely, for NMDA-mediated inputs, synaptic thresholds decreased with distance from the soma, or with depolarization of the RMP (Fig. $6 D$, blue plots). As was true in the CA3 neuron, the combined presence of synaptic AMPA and NMDA conductances in the dentate granule neuron minimized the impact of dendritic location and RMP on synaptic thresholds (Fig. $6 D$, green plots) and greatly reduced the CVs of thresholds calculated across RMPs for increasingly distant dendritic locations (Fig. $6 E$ ) or across dendritic location within a given RMP (Fig. 6F). Mean total CVs for the ten spatiotemporal input patterns, calculated across all dendritic locations and RMPs, were $0.19 \pm 0.02,0.52 \pm 0.01$, and $0.08 \pm 0.01$, for AMPA-only, NMDA-only, and AMPA-and-NMDA conductances, respectively, with an effect size of -5.18 $s_{\text {AMPA-only }}$ for AMPA+NMDA inputs relative to AMPA-only inputs.

\section{Impact of AMPA-to-NMDA conductance ratios on synaptic integration}

The simulations described above reveal that combining AMPA and NMDA conductances can increase the fidelity of synaptic integration, as measured at the soma and axon, across dendritic locations and/or RMPs. However, while the number of synaptic NMDA receptors is fairly uniform across most synapses (on the order of 10 receptors per synapse; Racca et al., 2000; Nimchinsky et al., 2004; Noguchi et al., 2005), synaptic AMPA conductances can range from zero (i.e., in "silent" synapses) to $>1.5 \mathrm{nS}$ (Beaulieu-Laroche and Harnett, 2018), and are dynamic in response to synaptic plasticity (for review, see Herring and Nicoll, 2016). Thus, the ratio of AMPA-to-NMDA conductance at synapses is variable. To determine the impact of AMPA-to-NMDA ratio on the fidelity of synaptic integration, we varied the maximal AMPA conductance from 0.1 to $1.5 \mathrm{nS}$ while keeping the maximal NMDA 


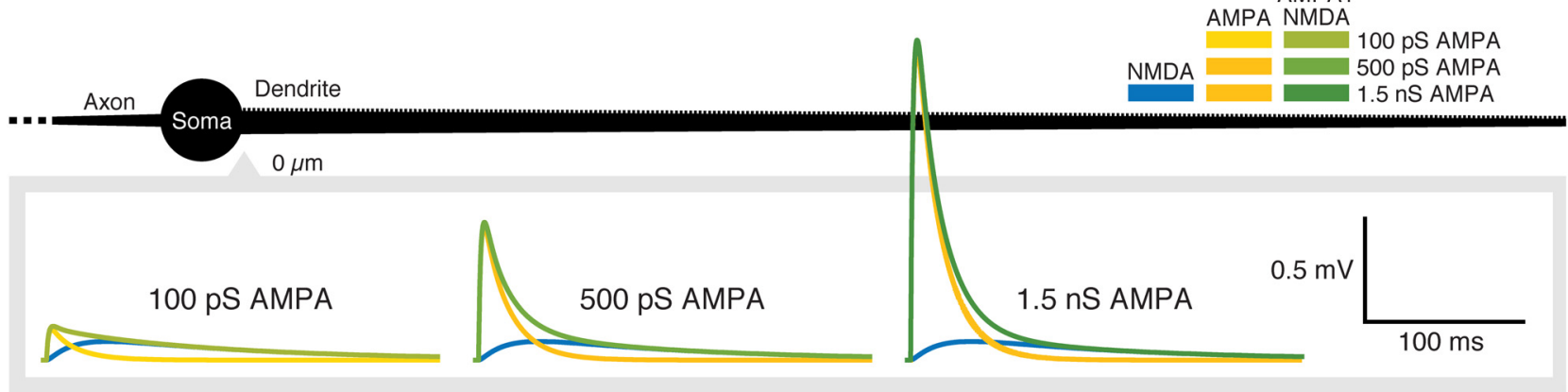

B
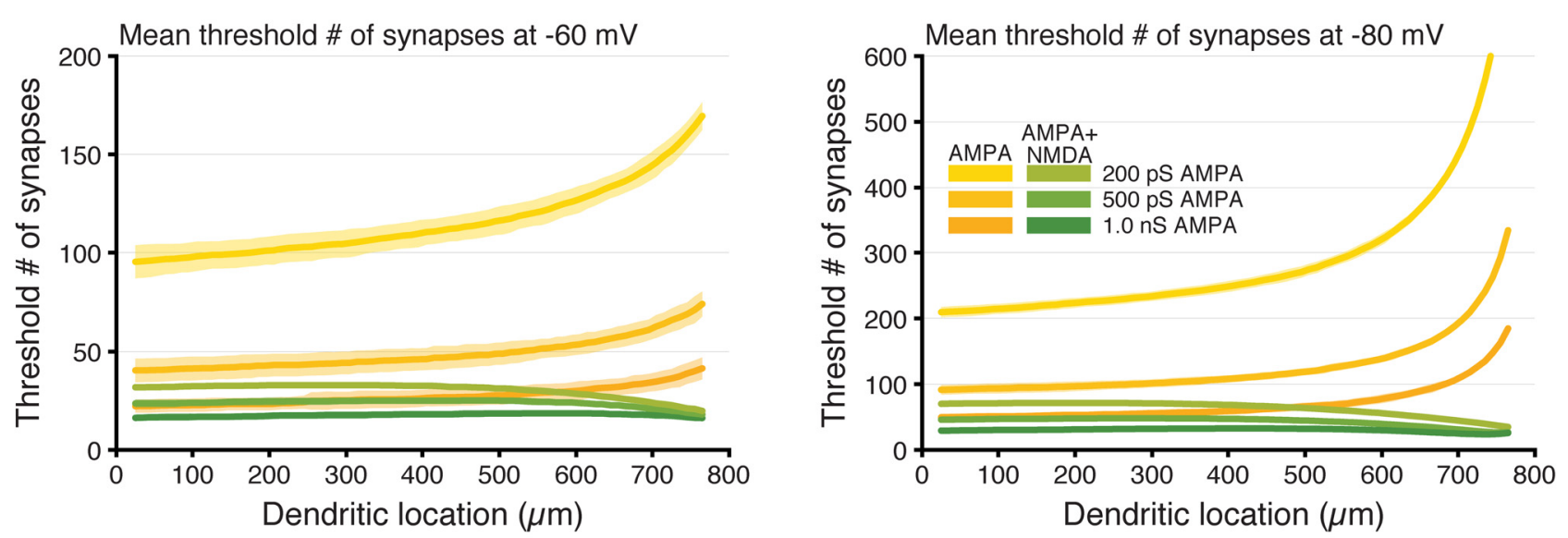

C

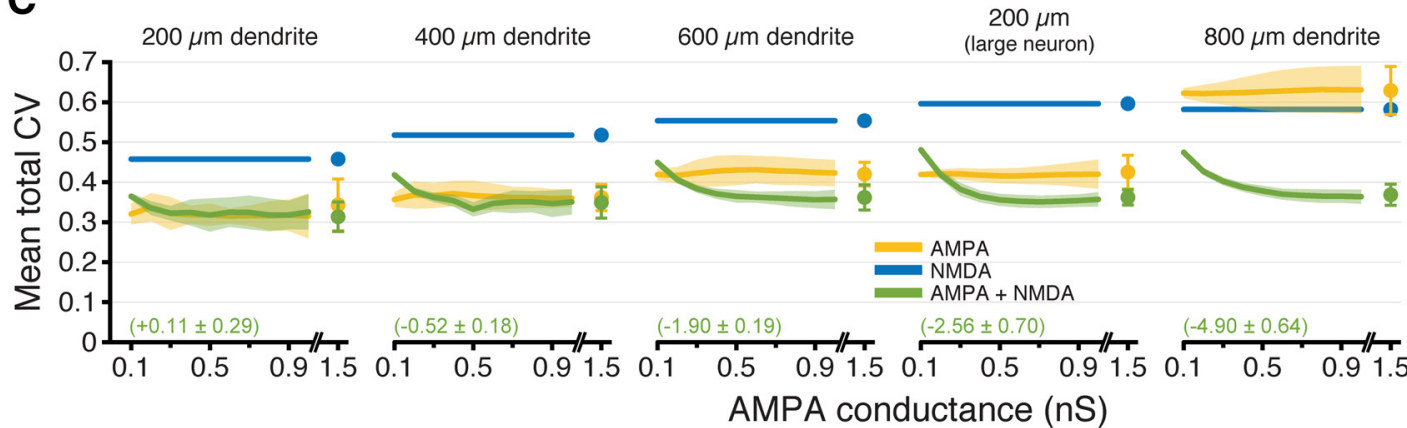

$200 \mu \mathrm{m}$

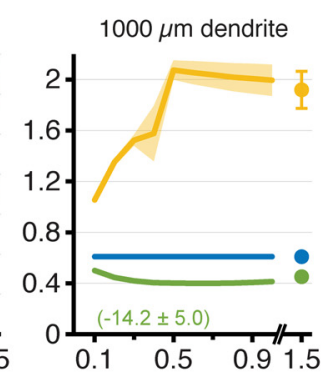

Figure 7. NMDA-dependent stabilization of EPSP-spike coupling occurs over a range of AMPA-to-NMDA conductance ratios. $\boldsymbol{A}$, Somatic EPSPs generated at $-70 \mathrm{mV}$ by proximal inputs having the indicated maximum AMPA conductances (maximal NMDA conductance held steady at $1 \mathrm{nS}$ ) in a ball-and-stick neuron (black; not drawn to scale). $\boldsymbol{B}$, Plots of the mean number of synaptic activations necessary to fire an action potential ( \pm SDs; shaded regions) in an $800-\mu \mathrm{m}$-long ball-and-stick neuron resting at $-60 \mathrm{mV}$ (left) or $-80 \mathrm{mV}$ (right) with peak AMPA conductance set to $0.2,0.5$, or $1 \mathrm{nS}$, either alone (yellow) or together with a $1 \mathrm{nS}$ NMDA conductance (green); $y$-axes limited to 200 (left) or 600 (right) synapses to show differences in proximal locations. C, Plots of mean CVs for thresholds ( \pm SDs; shaded regions) for each dendritic length (including the $200-\mu \mathrm{m}$ dendrite on a large neuron with three additional $600-\mu \mathrm{m}$ dendrites) for inputs having AMPA-only (yellow) or AMPA plus NMDA (green) conductances as a function of maximal AMPA conductance (0.1-1.5 nS). Mean effect sizes for AMPA+NMDA inputs (relative to AMPA-only inputs; \pm SDs) calculated for all AMPA conductances $\geq 400 \mathrm{pS}$ are shown in green. As a reference, the CV ( \pm SD) for NMDA-only synapses is shown in blue. Note the $y$ axis scale change for the $1000-\mu \mathrm{m}$ dendrite.

conductance steady at $1 \mathrm{nS}$, and measured the minimum number of synaptic activations necessary to trigger an action potential in ball-and-stick neurons of various dendritic lengths (Fig. 7). Examples of individual synaptic responses generated by AMPA-only and AMPA+NMDA inputs containing different magnitudes of AMPA conductance are shown in Figure 7A (NMDA-only inputs are also shown for comparison). Regardless of AMPA conductance magnitude, the threshold number of synaptic inputs for action potential initiation increased sharply with distance from the soma for AMPA-only inputs, while thresholds for inputs containing both AMPA and NMDA conductances were relatively independent of distance (Fig. 7B). In long dendrites (>600 $\mu \mathrm{m})$, the mean CV for the threshold number of inputs was lowest when both conductances were present, regardless of AMPA-toNMDA ratio (Fig. $7 C$ ). In ball-and-stick model neurons with short dendrites, when AMPA conductances were 


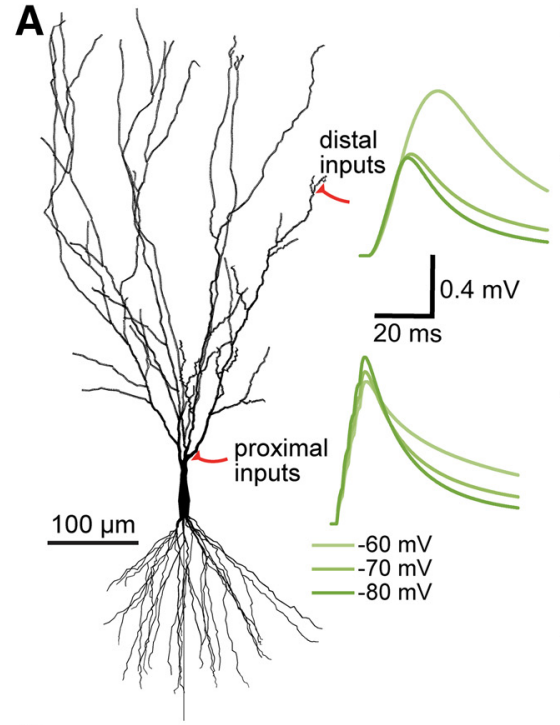

C

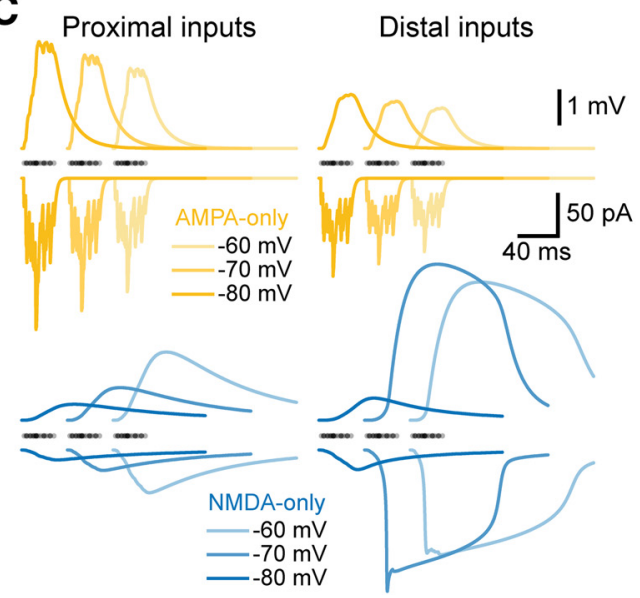

B Proximal inputs (AMPA + NMDA)

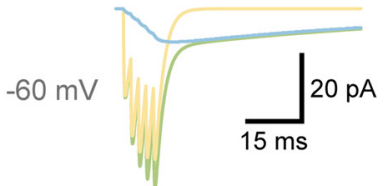

$-70 \mathrm{mV}$

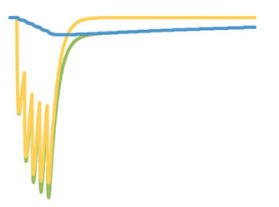

Distal inputs (AMPA + NMDA)

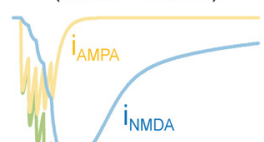

$-80 \mathrm{mV}$

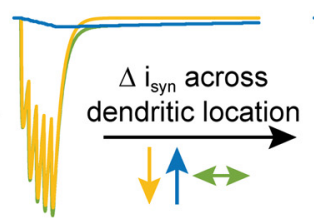

Proximal inputs

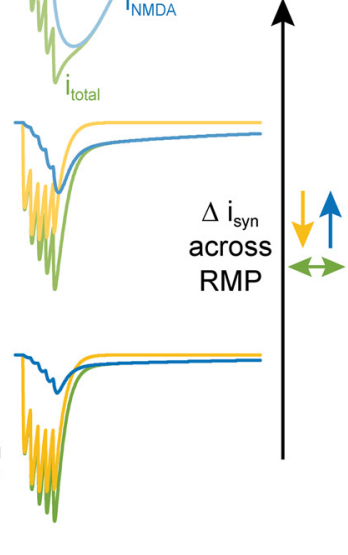

Distal inputs

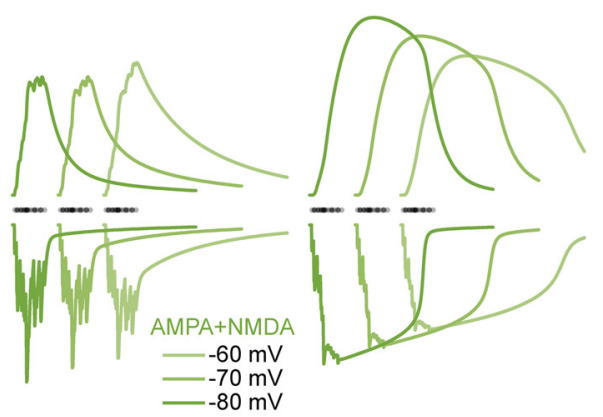

Figure 8. Summary of NMDA-dependent stabilization of synaptic integration across dendritic location and RMP. A, Morphology of a CA3 neuron indicating the locations of proximal and distal synaptic inputs to the apical dendrite. Traces at right show the somatic responses to sequential activation of five AMPA+NMDA synapses at neighboring spines (at 2-ms intervals, distal to proximal) at three different RMPs (as indicated by color depth) for each input location (distal vs proximal) in a fully passive model. $\boldsymbol{B}$, Synaptic currents ( $\left.\mathrm{i}_{\text {syn }}\right)$ generated by activation of the five proximal (left) or five distal (right) inputs at three RMPs. Total synaptic currents (itotal) are shown in green, with AMPA (i $\mathrm{i}_{\text {AMPA }}$; yellow) and NMDA (i $\mathrm{IMDA}_{\mathrm{NM}}$; blue) components superimposed. Notice that AMPA currents are reduced (yellow arrows), while NMDA currents are enhanced (blue arrows), as inputs are moved from proximal to distal locations, or as RMP is depolarized from -80 to $-60 \mathrm{mV}$. These opposing effects of location and RMP limit the variability of the total synaptic current across location and RMP (green arrows). $\boldsymbol{C}$, Similar to $\boldsymbol{B}$, but showing somatic voltage responses (upper traces) and summed synaptic currents (lower traces) for a barrage of 25 synaptic inputs with stochastic timings and spine locations for AMPA-only (yellow), NMDA-only (blue), or AMPA+NMDA (green) inputs. Timings of synapses indicated with semi-translucent dots above current traces. Note how AMPA-only currents and somatic EPSPs (yellow) get smaller with depolarization or distance from the soma, while NMDA-only currents and EPSPs (blue) get larger with distance and depolarization. Combining AMPA and NMDA conductance (green) leads to less variation in the amplitudes of both synaptic current and somatic EPSPs across RMPs and/or dendritic locations.

small, adding an NMDA conductance did not significantly reduce threshold CVs, as the NMDA conductance became the dominant driver of somatic depolarization, and CVs converged toward those of NMDA-only inputs. This reflects the tight electrotonic structure of small model neurons, as adding NMDA conductance reduced the CV of EPSP-spike coupling across most AMPA-to-NMDA ratios in the 200- $\mu \mathrm{m}$ dendrite when it was attached to a larger neuron with three additional $600-\mu \mathrm{m}$ dendrites ("large neuron" in Fig. 7C; compare also results from the short-but-branched dendritic arbor of the dentate granule neuron in Fig. 6D-F). For all AMPA conductances $\geq 400$ pS, the mean effect size of adding NMDA conductance (relative to AMPA-only inputs) varied between negligible $\left(d=+0.11 \pm 0.29 s_{\text {AMPA-only }}\right.$ in the $200-\mu \mathrm{m}$ dendrite) to enormous $\left(d=-15.2 \pm 5.0 s_{\text {AMPA-only }}\right.$ in the $1000-\mu \mathrm{m}$ dendrite), with mean effect sizes always being more negative than -1.0 for dendrites $\geq 600 \mu \mathrm{m}$ (including in the 200- $\mu \mathrm{m}$ dendrite attached to a larger neuron; Fig. 7C). Thus, combining AMPA and NMDA conductances enhances the fidelity of synaptic integration across a wide range of physiologically relevant AMPA-to-NMDA ratios. 


\section{Discussion}

Synaptic integration, the process by which patterns of synaptic input are transduced into action potential initiation (also known as "EPSP-spike coupling"), is the core of neural computation. We tested the impact of two prominent and often coexpressed glutamate receptor subtypes, AMPA and NMDA receptors, on the fidelity of synaptic integration across dendritic location and neuronal state (i.e., initial membrane potential). Our results demonstrate that the kinetics and voltage dependence of the NMDA conductance act synergistically to counterbalance the impact of distance-dependent signal attenuation and the reduced synaptic driving forces occurring at depolarized membrane potentials, effectively increasing the fidelity of EPSP-spike coupling across both spatial and voltage domains (as summarized in Fig. 8). This intrinsic consequence of combining synaptic AMPA and NMDA receptors occurs over a broad range of neuron morphologies and AMPA-to-NMDA conductance ratios, and is independent of, but fully compatible with, its well characterized role in gating synaptic plasticity.

\section{Interaction of electrotonic structure and synaptic conductance}

EPSPs are shaped by the electrotonic structure of the dendritic tree; in narrow distal dendrites, small surface areas limit local membrane conductance and capacitance, yielding greater input impedance and larger and faster local EPSPs in response to synaptic currents (Gulledge et al., 2005). This can reduce synaptic currents in distal dendrites, as greater local depolarization during EPSPs limits synaptic driving forces at the peak of the EPSP. At the same time, dendrites act as low-pass filters for EPSPs spreading from the site of synaptic input toward the soma. Thus, in the absence of voltage-dependent conductances, the somatic efficacy of synaptic input diminishes with distance from the soma, thereby requiring a greater number of synaptic inputs to reach action potential threshold. Our results with AMPA-only synaptic inputs are fully consistent with these well-described dendritic cable properties (Figs. 1, 2). NMDA conductances, on the other hand, generate larger synaptic currents in distal, high impedance dendritic compartments because of their enhanced conductance at depolarized membrane potentials (Gulledge et al., 2012; Lajeunesse et al., 2013). Our main result is that the combined presence of AMPA and NMDA receptors intrinsically lowers location-dependent and RMP-dependent variability in synaptic efficacy by boosting synaptic conductance preferentially in high-impedance dendritic compartments (Branco and Häusser, 2011; Gulledge et al., 2012) and/or when neurons are in depolarized states (Branco et al., 2010), conditions in which synaptic driving forces are diminished (see Figs. 2, 8). NMDA-dependent stabilization of synaptic integration was robust across neuron morphologies (Figs. 2, 3, 6) and over a wide range of AMPA-toNMDA conductance ratios (Fig. 7), and persisted in the presence of active properties in dendrites (Fig. 3C) that can contribute to voltage-dependent amplification of inward current following synaptic activation (Magee and Johnston, 1995; Lipowsky et al., 1996; Branco et al., 2010).
By doping the AMPA conductance with voltage dependence (fast-NMDA) or slower kinetics (slow-AMPA; see Figs. $4,5)$, we were able to measure the relative impact of these two distinct properties of the NMDA conductance. Adding voltage dependence to the AMPA conductance reduced the variability of synaptic thresholds for action potential generation when measured across dendritic location within a given RMP, especially in long dendrites, but generally increased variability of synaptic thresholds when measured across RMP at a given dendritic location. This reflects the gradual recruitment of the NMDA conductance as synaptic barrages occur in progressively more-distal higher-impedance locations, leading to larger distal synaptic currents (see Figs. 2B, 8) and therefore fewer synaptic activations to reach threshold. On the other hand, the increased variability of EPSP-spike coupling when barrages were initiated at different RMPs reflects the nonlinear amplification of synaptic responses with increased depolarization, leading to a greater range of synaptic thresholds across RMPs. The effects of the fast-NMDA conductance on synaptic threshold variability across RMPs is greater in proximal dendritic locations for two reasons. First, voltage-dependent amplification is limited in distal, high-impedance compartments because EPSPs (including NMDA-spikes) approach the synaptic equilibrium potential. Second, AMPA-only inputs exhibit larger and faster exponential increases in synaptic thresholds with distance from the soma (see Fig. 4B). Together, these processes likely explain why voltage dependence alone (i.e., the fast-NMDA conductance) generates relatively higher CVs across RMPs at proximal dendritic locations, but lower CVs across RMPs in distal dendrites.

The slower kinetics of NMDA receptors, on their own, reduced the variability of synaptic thresholds selectively across RMP at proximal dendritic locations by increasing the opportunity for temporal summation during barrages. At any point within the stochastic barrage, the next iterative input is more likely to summate to a suprathreshold response if EPSP decay is slow. Since the decay of EPSPs depends on the local time constant, which itself is location dependent (i.e., larger near the soma; compare, for instance, the decay rates of proximal vs distal EPSPs having identical kinetics in Fig. 1A), the impact of slower kinetics will be largest in proximal dendritic locations (see Figs. 4, 5). The overall impact of adding voltage-dependent and slower NMDA conductances to AMPA synapse is to greatly reduce the variability of somatic responses and EPSP-spike coupling across both dendritic location and membrane potential state (Figs. 2, 6).

\section{Impact of NMDA conductance on synaptic integration and network performance}

Neurons employ a variety of mechanisms to combat location-dependent variability in synaptic efficacy. In the apical dendrites of CA1 pyramidal neurons, synaptic conductance is scaled in proportion to distance from the soma to minimize location-dependent variability of axonal drive (Magee and Cook, 2000; Nicholson et al., 2006; Shipman et al., 2013). Alternatively, dendrites may express dendritic voltage-gated sodium and calcium 
conductances that, with sufficient local depolarization, can generate dendritic action potentials that amplify somatic EPSPs (Magee et al., 1995; Magee and Johnston, 1995; Schiller et al., 1997; Golding and Spruston, 1998; Golding et al., 1999; Milojkovic et al., 2005). Similarly, synaptic NMDA receptors, when activated in sufficient numbers, generate self-sustaining plateau potentials (NMDA spikes) that lead to supralinear synaptic summation and larger somatic EPSPs (Schiller et al., 2000; Wei et al., 2001; Polsky et al., 2004; Nevian et al., 2007; Major et al., 2008; Larkum et al., 2009; Branco et al., 2010; Branco and Häusser, 2011; Harnett et al., 2012). These voltage-dependent synaptic and dendritic mechanisms act preferentially in distal dendritic compartments where input impedance is highest to facilitate synaptic depolarization of the soma and axon despite distance-dependent voltage-attenuation within the dendritic arbor. However, unlike action potentials, NMDA spikes are not all-or-none. Instead, they vary in amplitude and duration in proportion to the number of activated synapses, the local input impedance at the synapse, and the initial membrane potential (Major et al., 2008; Branco et al., 2010; Branco and Häusser, 2011; Gulledge et al., 2012; Farinella et al., 2014). Thus, although highly nonlinear across voltage, NMDA spikes are effectively "graded" in amplitude and duration across dendritic locations (see Fig. $2 B$ ), allowing the NMDA conductance to enhance synaptic current in proportion to electrotonic distance from the soma and axon. Similarly, by opposing the impact of reduced synaptic driving force on AMPA-mediated currents, voltage-dependent NMDA conductances can reduce the variability of total synaptic current during temporal integration of clustered excitatory inputs (Connelly et al., 2016; see also Fig. 8C).

Over the past several decades, there has been growing appreciation that, in addition to gating many forms of synaptic plasticity, NMDA receptors play an integral role in normal synaptic integration (for review, see Häusser and Mel, 2003; Antic et al., 2010; Major et al., 2013). In the distal apical tufts of layer 5 neurons, where high input impedance favors amplification of local EPSPs, NMDA activation boosts synaptic currents such that they can more reliably trigger calcium spikes at an electrically excitable zone at the base of the tuft (Larkum et al., 2009). Indeed, simulations by Larkum et al. (2009) found the threshold number of synapses necessary for initiating an apical trunk calcium spike to be relatively consistent across tuft locations when NMDA conductances were present, but that thresholds for AMPA-only inputs grew exponentially, and to non-physiological synaptic densities, with distance from the initiation zone in the apical dendrite. Thus, the impact of NMDA receptors on the fidelity of EPSP-spike coupling may not be limited to action potentials initiated at the axon, but likely applies more generally for spike initiation at any specialized trigger zone, so long as it is electrotonically downstream (e.g., in a larger compartment) from the site of synaptic input.

In conclusion, transduction of synaptic events into patterns of action potential output is the most fundamental neuronal task. It is a process influenced by the strength and kinetics of individual synaptic conductances, the spatiotemporal relationships among them, and the electrotonic properties of the neuron. Nonlinear amplification of synaptic input via NMDA spikes is proposed to increase the "computational power" of neurons (Wei et al., 2001; Poirazi et al., 2003; Branco and Häusser, 2011). Our results demonstrate that NMDA receptors, via their intrinsic kinetics and voltage dependence, also provide the advantage of "computational stability" by enhancing the fidelity of EPSP-spike coupling across dendritic domains and membrane potential states. While speculative, it is interesting to consider whether this consequence of NMDA receptor expression may have provided advantages to primitive nervous systems (e.g., in cnidaria; Anctil, 2009; Pierobon, 2012) independent of their role in associative plasticity, which may account for their ancient evolutionary origin in the common ancestor of all metazoans (Ramos-Vicente et al., 2018).

\section{References}

Anctil M (2009) Chemical transmission in the sea anemone Nematostella vectensis: a genomic perspective. Comp Biochem Physiol Part D Genomics Proteomics 4:268-289.

Antic SD, Zhou WL, Moore AR, Short SM, Ikonomu KD (2010) The decade of the dendritic NMDA spike. J Neurosci Res 88:29913001.

Beaulieu-Laroche L, Harnett MT (2018) Dendritic spines prevent synaptic voltage clamp. Neuron 97:75-82.e3.

Branco T, Häusser M (2010) The single dendritic branch as a fundamental functional unit in the nervous system. Curr Opin Neurobiol 20:494-502.

Branco T, Häusser M (2011) Synaptic integration gradients in single cortical pyramidal cell dendrites. Neuron 69:885-892.

Branco T, Clark BA, Häusser M (2010) Dendritic discrimination of temporal input sequences in cortical neurons. Science 329:16711675.

Carnevale NT, Hines ML (2006) The NEURON book. New York: Cambridge University Press.

Cash S, Yuste R (1998) Input summation by cultured pyramidal neurons is linear and position-independent. J Neurosci 18:10-15.

Cichon J, Gan WB (2015) Branch-specific dendritic $\mathrm{Ca}(2+)$ spikes cause persistent synaptic plasticity. Nature 520:180-185.

Connelly WM, Crunelli V, Errington AC (2016) Passive synaptic normalization and input synchrony-dependent amplification of cortical feedback in thalamocortical neuron dendrites. J Neurosci 36:3735-3754.

Diamond JS, Copenhagen DR (1993) The contribution of NMDA and non-NMDA receptors to the light-evoked input-output characteristics of retinal ganglion cells. Neuron 11:725-738.

Farinella M, Ruedt DT, Gleeson P, Lanore F, Silver RA (2014) Glutamate-bound NMDARs arising from in vivo-like network activity extend spatio-temporal integration in a L5 cortical pyramidal cell model. PLoS Comput Biol 10:e1003590.

Golding NL, Spruston N (1998) Dendritic sodium spikes are variable triggers of axonal action potentials in hippocampal CA1 pyramidal neurons. Neuron 21:1189-1200.

Golding NL, Jung HY, Mickus T, Spruston N (1999) Dendritic calcium spike initiation and repolarization are controlled by distinct potassium channel subtypes in CA1 pyramidal neurons. J Neurosci 19:8789-8798.

Greer JB, Khuri S, Fieber LA (2017) Phylogenetic analysis of ionotropic L-glutamate receptor genes in the bilateria, with special notes on Aplysia californica. BMC Evol Biol 17:11.

Grienberger C, Chen X, Konnerth A (2014) NMDA receptor-dependent multidendrite $\mathrm{Ca}(2+)$ spikes required for hippocampal burst firing in vivo. Neuron 81:1274-1281.

Grienberger C, Chen X, Konnerth A (2015) Dendritic function in vivo. Trends Neurosci 38:45-54. 
Gulledge AT, Kampa BM, Stuart GJ (2005) Synaptic integration in dendritic trees. J Neurobiol 64:75-90.

Gulledge AT, Carnevale NT, Stuart GJ (2012) Electrical advantages of dendritic spines. PLoS One 7:e36007.

Harnett MT, Makara JK, Spruston N, Kath WL, Magee JC (2012) Synaptic amplification by dendritic spines enhances input cooperativity. Nature 491:599-602.

Häusser M, Mel B (2003) Dendrites: bug or feature? Curr Opin Neurobiol 13:372-383.

Hemond P, Migliore M, Ascoli GA, Jaffe DB (2009) The membrane response of hippocampal CA3b pyramidal neurons near rest: heterogeneity of passive properties and the contribution of hyperpolarizationactivated currents. Neuroscience 160:359-370.

Herring BE, Nicoll RA (2016) Long-term potentiation: from CaMKII to AMPA receptor trafficking. Annu Rev Physiol 78:351-365.

lacobucci GJ, Popescu GK (2017) NMDA receptors: linking physiological output to biophysical operation. Nat Rev Neurosci 18:236249.

Jaffe DB, Carnevale NT (1999) Passive normalization of synaptic integration influenced by dendritic architecture. J Neurophysiol 82:3268-3285.

Lajeunesse F, Kröger H, Timofeev I (2013) Regulation of AMPA and NMDA receptor-mediated EPSPs in dendritic trees of thalamocortical cells. J Neurophysiol 109:13-30.

Larkum ME, Nevian T, Sandler M, Polsky A, Schiller J (2009) Synaptic integration in tuft dendrites of layer 5 pyramidal neurons: a new unifying principle. Science 325:756-760.

Lipowsky R, Gillessen T, Alzheimer C (1996) Dendritic Na+ channels amplify EPSPs in hippocampal CA1 pyramidal cells. J Neurophysiol 76:2181-2191.

Lüscher C, Malenka RC (2012) NMDA receptor-dependent longterm potentiation and long-term depression (LTP/LTD). Cold Spring Harb Perspect Biol 4:a005710.

Magee JC, Johnston D (1995) Synaptic activation of voltage-gated channels in the dendrites of hippocampal pyramidal neurons. Science 268:301-304.

Magee JC, Cook EP (2000) Somatic EPSP amplitude is independent of synapse location in hippocampal pyramidal neurons. Nat Neurosci 3:895-903.

Magee JC, Christofi G, Miyakawa H, Christie B, Lasser-Ross N, Johnston D (1995) Subthreshold synaptic activation of voltagegated $\mathrm{Ca} 2+$ channels mediates a localized $\mathrm{Ca} 2+$ influx into the dendrites of hippocampal pyramidal neurons. J Neurophysiol 74:1335-1342.

Major G, Polsky A, Denk W, Schiller J, Tank DW (2008) Spatiotemporally graded NMDA spike/plateau potentials in basal dendrites of neocortical pyramidal neurons. J Neurophysiol 99:2584-2601.

Major G, Larkum ME, Schiller J (2013) Active properties of neocortical pyramidal neuron dendrites. Annu Rev Neurosci 36:1-24.

Milojkovic BA, Wuskell JP, Loew LM, Antic SD (2005) Initiation of sodium spikelets in basal dendrites of neocortical pyramidal neurons. J Membr Biol 208:155-169.

Nevian T, Larkum ME, Polsky A, Schiller J (2007) Properties of basal dendrites of layer 5 pyramidal neurons: a direct patch-clamp recording study. Nat Neurosci 10:206-214.
Nicholson DA, Trana R, Katz Y, Kath WL, Spruston N, Geinisman Y (2006) Distance-dependent differences in synapse number and AMPA receptor expression in hippocampal CA1 pyramidal neurons. Neuron 50:431-442.

Nimchinsky EA, Yasuda R, Oertner TG, Svoboda K (2004) The number of glutamate receptors opened by synaptic stimulation in single hippocampal spines. J Neurosci 24:2054-2064.

Noguchi J, Matsuzaki M, Ellis-Davies GC, Kasai H (2005) Spine-neck geometry determines NMDA receptor-dependent $\mathrm{Ca} 2+$ signaling in dendrites. Neuron 46:609-622.

Palmer LM, Shai AS, Reeve JE, Anderson HL, Paulsen O, Larkum ME (2014) NMDA spikes enhance action potential generation during sensory input. Nat Neurosci 17:383-390.

Pierobon P (2012) Coordinated modulation of cellular signaling through ligand-gated ion channels in Hydra vulgaris (cnidaria, hydrozoa). Int J Dev Biol 56:551-565.

Poirazi P, Brannon T, Mel BW (2003) Pyramidal neuron as two-layer neural network. Neuron 37:989-999.

Polsky A, Mel BW, Schiller J (2004) Computational subunits in thin dendrites of pyramidal cells. Nat Neurosci 7:621-627.

Polsky A, Mel B, Schiller J (2009) Encoding and decoding bursts by NMDA spikes in basal dendrites of layer 5 pyramidal neurons. $J$ Neurosci 29:11891-11903.

Racca C, Stephenson FA, Streit P, Roberts JD, Somogyi P (2000) NMDA receptor content of synapses in stratum radiatum of the hippocampal CA1 area. J Neurosci 20:2512-2522.

Ramos-Vicente D, Ji J, Gratacòs-Batlle E, Gou G, Reig-Viader R, Luís J, Burguera D, Navas-Perez E, García-Fernández J, FuentesPrior P, Escriva H, Roher N, Soto D, Bayés À (2018) Metazoan evolution of glutamate receptors reveals unreported phylogenetic groups and divergent lineage-specific events. Elife 7:e35774.

Schiller J, Schiller Y, Stuart G, Sakmann B (1997) Calcium action potentials restricted to distal apical dendrites of rat neocortical pyramidal neurons. J Physiol 505:605-616.

Schiller J, Major G, Koester HJ, Schiller Y (2000) NMDA spikes in basal dendrites of cortical pyramidal neurons. Nature 404:285289.

Schmidt-Hieber C, Jonas P, Bischofberger J (2007) Subthreshold dendritic signal processing and coincidence detection in dentate gyrus granule cells. J Neurosci 27:8430-8441.

Shipman SL, Herring BE, Suh YH, Roche KW, Nicoll RA (2013) Distance-dependent scaling of AMPARs is cell-autonomous and GluA2 dependent. J Neurosci 33:13312-13319.

Sivagnanam S, Majumdar A, Yoshimoto K, Astakhov V, Bandrowski A, Martone ME, Carnevale NT (2013) Introducing the neuroscience gateway. In: International workshop on science gateways (Kiss T, ed). Zürich: CEUR-WS.org.

Stern P, Edwards FA, Sakmann B (1992) Fast and slow components of unitary EPSCs on stellate cells elicited by focal stimulation in slices of rat visual cortex. J Physiol 449:247-278.

Wei DS, Mei YA, Bagal A, Kao JP, Thompson SM, Tang CM (2001) Compartmentalized and binary behavior of terminal dendrites in hippocampal pyramidal neurons. Science 293:2272-2275. 